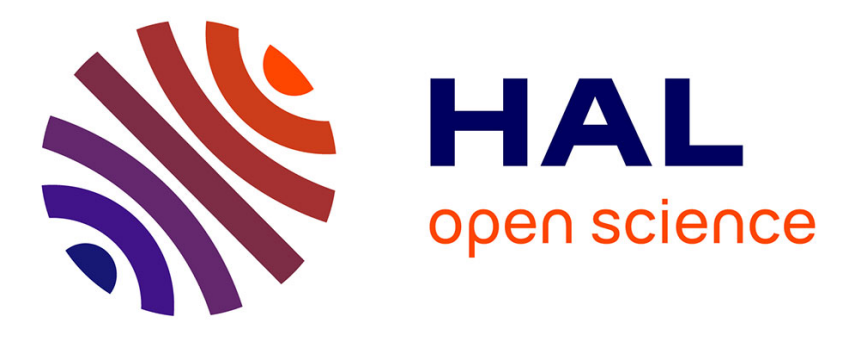

\title{
SOA Antipatterns: an Approach for their Specification and Detection
}

\author{
Palma Francis, Nayrolles Mathieu, Moha Naouel, Guéhéneuc \\ Yann-Gael.Gueheneuc@polymtl.Ca Yann-Gael, Benoit Baudry, Jean-Marc \\ Jézéquel
}

\section{To cite this version:}

Palma Francis, Nayrolles Mathieu, Moha Naouel, Guéhéneuc Yann-Gael.Gueheneuc@polymtl.Ca Yann-Gael, Benoit Baudry, et al.. SOA Antipatterns: an Approach for their Specification and Detection. International Journal of Cooperative Information Systems, 2013. hal-00916299

\section{HAL Id: hal-00916299 \\ https://hal.inria.fr/hal-00916299}

Submitted on 10 Dec 2013

HAL is a multi-disciplinary open access archive for the deposit and dissemination of scientific research documents, whether they are published or not. The documents may come from teaching and research institutions in France or abroad, or from public or private research centers.
L'archive ouverte pluridisciplinaire HAL, est destinée au dépôt et à la diffusion de documents scientifiques de niveau recherche, publiés ou non, émanant des établissements d'enseignement et de recherche français ou étrangers, des laboratoires publics ou privés. 


\section{International Journal of Cooperative Information Systems SOA ANTIPATTERNS: AN APPROACH FOR THEIR SPECIFICATION AND DETECTION \\ --Manuscript Draft--}

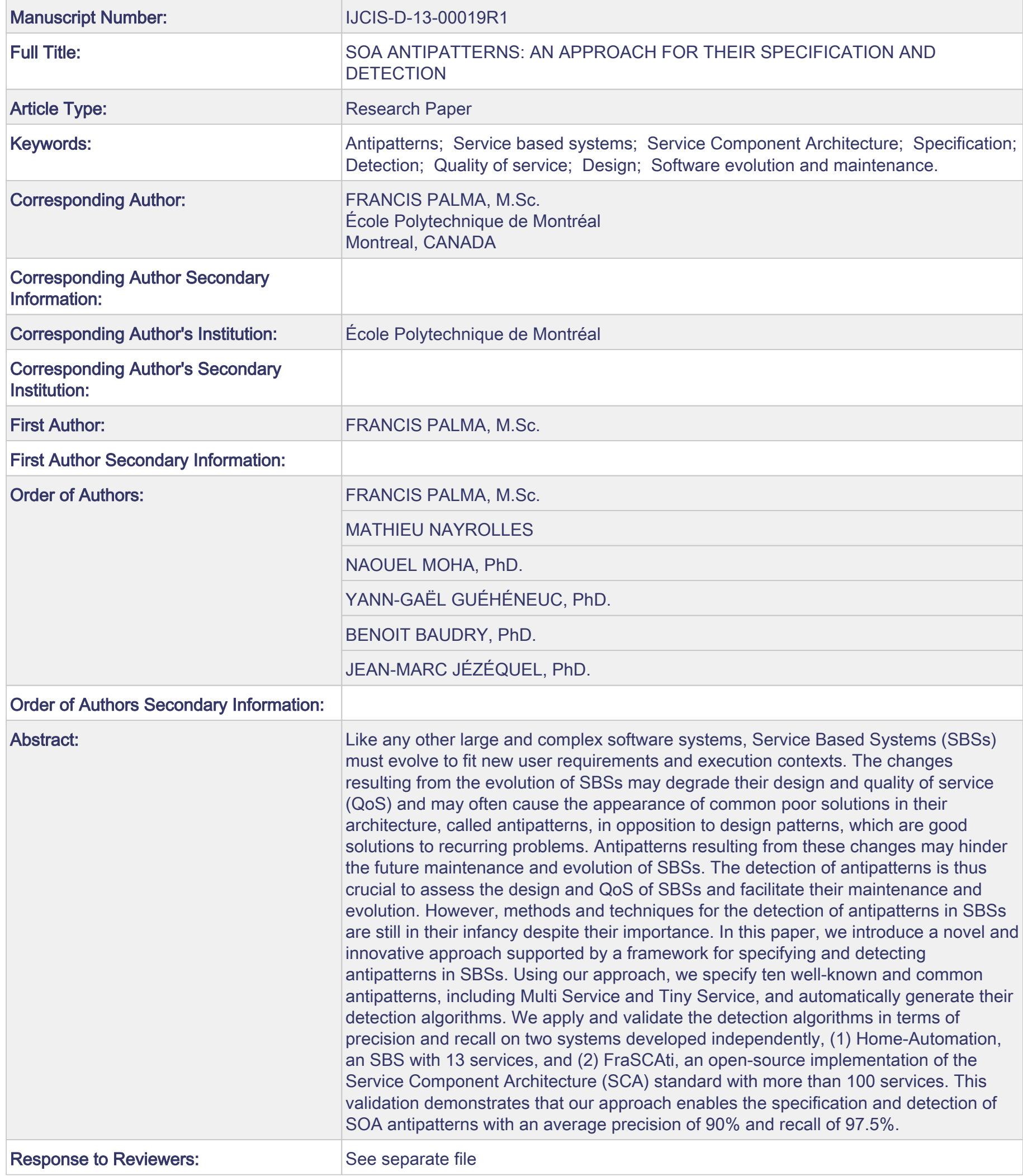




\section{Response to the Reviewers Comments}

SOA Antipatterns: An Approach for their Specification and Detection Francis Palma, Mathieu Nayrolles, Naouel Moha, Yann-Gaël Guéhéneuc, Benoit Baudry, Jean-Marc Jézéquel

\section{Introduction}

We would like to thank the reviewers for their detailed and useful comments. We have undertaken a revision of the work, taking into account and acting on these comments. In the following, we explain how we addressed the concerns expressed by the reviewers. For better readability, reviewers comments appear in italic while our answers appear in normal style.

\section{Reviewer Comments}

\section{Reviewer 1}

[R1-1] "Is it possible to study the cause of false positive detection?"

Thanks for pointing out this to our attention. We missed to discuss this important aspect. We have added two paragraphs inside the Section 4.5.2 and Section 4.5.4 to discuss the false positive detections for our two experiments. For Home-Automation, we have discussed two the cause of two false positives in Section 4.5.2. As for FraS$C A t i$, we have discussed the same for three antipatterns in Section 4.5.4.

[R1-2] "Is there any planned action toward reducing the false positive detection?" Indeed, this is an significant aspect to improve precision. Even for a high number of services, we have considerable precision, i.e., for Home-Automation we have a precision of $92.5 \%$ and for FraSCAti we have $89 \%$ as the precision with more than 100 of services. We also discussed in Section 4.5.2 and Section 4.5.4 our actions to reduce the false positives.

[R1-3] "The authors said "Such low computation times suggest that SODA could be applied on SBSs even with a larger number of services", so on which approximately number of services the approach can't be applied?"

Thanks for this insightful comments. Indeed, our approach, SODA is applicable for SBS with a low to a high number of services, i.e., from 13 to more than 100 
as shown with our experiments. Interestingly, the detection times were fraction of seconds for Home-Automation and FraSCAti. The detection times depend mostly on computing some static metrics, such as NMD, CPL, COH, or ANIM. Computing those metrics requires thorough inspection of source code and sometimes a high number of pairwise comparisons among method signatures and/or parameters. However, the complexity of our detection algorithms are linear, i.e., $O(\mathrm{n})$ with n represents the number of rules. Therefore, the cumulative detection times will increase with the number of rules in each rule card, and with the number of antipatterns to be detected in the target system.

\section{Reviewer 2}

[R2-1] "While the experimental results are presented and analyzed in detail, the delta is not very significant and would not bring much more information for a reader of the ICSOC paper."

We tried to explain our contributions over the ICSOC paper in the Introduction section. We revised the related work section, made an extensive validation with detailed discussions, extended the DSL and specify three new antipatterns and performed their detection as well.

[R2-2] "From a presentation point of view, why the new patterns are inserted in the section "Discussion on the Assumptions"? They should be presented in the same section as the others (while the remark on extensibility could reference them)."

Thanks for identifying this presentation issue. We have reorganised the contents according to the suggestions. We moved the list of antipatterns (Table 1), the rule cards for different antipatterns (Figure 8), the list of three new antipatterns (Table 2 ), and the rule cards for three new antipatterns (Figure 9), all to Section 4.2.

[R2-3] "The approach itself should be presented in a more detailed way (the authors should use the extra space of a journal paper to better explain it): - the set operators (difference, inclusion, etc) and their use."

We thank the reviewer for pointing out this gap. We describe those operators in Section 3.1, where we explain our DSL and provide some examples in a new paragraph. Currently, the antipatterns specified for our experiments do not require for example DIFF or NEG operators. However, we intend to specify more antipatterns from the literature in that case these operators may be helpful. We also plan to conduct experiments with those newly specified antipatterns.

[R2-4] "The approach itself should be presented in a more detailed way (the authors 
should use the extra space of a journal paper to better explain it): - how Kermeta is used for generating the detection algorithms."

We thank the reviewer. We apologize for missing this point in our first version. We have included an example of the generation process of detection algorithms with Ecore and Acceleo in Section 3.2 to show how it works for our case. In our previous version of the implementation, we used Kermeta as meta-modeling framework support. In this version, we have changed our SOFA framework with more recent Ecore and Acceleo- meta-modeling facility. Ecore and Acceleo provide a more flexible way to handle and validate our DSL, rule cards, and the process of the generation of algorithms.

[R2-5] "The approach itself should be presented in a more detailed way (the authors should use the extra space of a journal paper to better explain it): - the computation of metrics that call sensors and triggers (examples could help also)"

We thank the reviewer. Added a small paragraph at the end of in Section 3.3 and explain how we use sensors and triggers to compute metrics with a small example.

[R2-6] "In the discussion of experimental results, the authors assume that the reader knows FraSCAti services and also their Home-Automation SBS."

We apologize for this strong assumption. Thanks for noticing this point. For the readers who are interested to know about services in Home-Automation and FraS$C A t i$, we added the reference article in our bibliography. Also, we have added the URLs on the design and implementation details for both FraSCAti and HomeAutomation.

\section{Reviewer 3}

[R3-1] "I miss some industrial tools in this presentation of existing approaches, because tools like PMD or others provide some information. I would find interesting to at least referencing some of these tools."

We thank the reviewer; we missed in our paper to name some useful tools that can detect antipatterns and smells in object-oriented systems. We have revised the related work and add a few discussion. Indeed, there are several tools available in the literature provided by the industry and the academic community, such as cbsdetector, FindBugs, iPlasma, JDeodorant, PatOMat, PMD, SonarQube, SPARSE, etc.

[R3-2] "I would also add a precise definition of SBS. From my point of view, ser- 
vices as they are understood in the paper are in-house (internal) services (seen as components)."

We thank the reviewer. We apologize to have missed to introduce the definition of service-based systems. We added a definition in the Introduction section. As for the second point, in this paper, we experimented with SCA applications. Therefore, as the building blocks of SCA applications, components are the primary target of our analysis. Each component necessarily provides one service implementing one interface. Thus, a service here is an internal or in-house entity represented by a component.

[R3-3] "In order to get an optimal result from the framework, precise information are required on the services to be monitored. It is not the case for external services. I would understand services more as components. In fact, in the context of the paper, services must be controlled in order to get interesting results from the defined metrics."

Indeed, the precise information that we have with the experimented systems are: (i) the services interfaces like the signatures and (ii) the references to other services. In our experiments, we considered components as the building blocks of the servicebased systems (e.g., an SCA system), and components are local for our experiments. This facilitate us to own and control the components because we also have the local implementations for those components. However, for the components that cannot be controlled, i.e., external ones, involve some major challenges for analysing them statically and dynamically. We are currently solving this issue by extending our approach SODA and by adapting our framework SOFA.

[R3-4] "In fact, in the context of the paper, services must be controlled in order to get interesting results from the defined metrics."

We thank the reviewer. We are currently working on other approaches to analyse systems without any intrusion or control, i.e., without owning the components. One of the way might be using the execution traces of SBSs. Execution traces provide all the services' interactions and message details, and a blueprint of the target system's architecture. But the issue of possessing the ownership is a common problem and the main weak-point for any approaches that deal with entities owned by third parties.

[R3-5] "I think I would suggest to the authors to use the term component rather than service, and SCA rather than SOA."

We thank the reviewer. Our main goal is to focus on SOA antipatterns. SCA, being only a technology to implement SOA is the first step we investigated. However, we 
also plan to investigate other types of antipatterns, such as related to Web service, REST, BPEL processes, etc., which collectively provide the basis for SOA antipatterns. 


\title{
SOA ANTIPATTERNS: AN APPROACH FOR THEIR SPECIFICATION AND DETECTION
}

\author{
FRANCIS PALMA \\ Ptidej Team, DGIGL, École Polytechnique de Montréal, C.P. 6079, Succ. Centre-ville \\ Montréal, Quebec, H3C 3A7, Canada \\ Département d'informatique, Université du Québec à Montréal, C.P. 8888, Succ. Centre-ville \\ Montréal, Quebec, H3C 3P8, Canada \\ MATHIEU NAYROLLES AND NAOUEL MOHA \\ Département d'informatique, Université du Québec à Montréal, C.P. 8888, Succ. Centre-ville \\ Montréal, Quebec, H3C 3P8, Canada \\ YANN-GAËL GUÉHÉNEUC \\ Ptidej Team, DGIGL, École Polytechnique de Montréal, C.P. 6079, Succ. Centre-ville \\ Montréal, Quebec, H3C 3A7, Canada \\ BENOIT BAUDRY AND JEAN-MARC JÉZÉQUEL \\ INRIA Rennes, Université Rennes 1 \\ Rennes, France \\ Received (Day Month Year) \\ Revised (Day Month Year)
}

\begin{abstract}
Like any other large and complex software systems, Service Based Systems (SBSs) must evolve to fit new user requirements and execution contexts. The changes resulting from the evolution of SBSs may degrade their design and quality of service (QoS) and may often cause the appearance of common poor solutions in their architecture, called antipatterns, in opposition to design patterns, which are good solutions to recurring problems. Antipatterns resulting from these changes may hinder the future maintenance and evolution of SBSs. The detection of antipatterns is thus crucial to assess the design and QoS of SBSs and facilitate their maintenance and evolution. However, methods and techniques for the detection of antipatterns in SBSs are still in their infancy despite their importance. In this paper, we introduce a novel and innovative approach supported by a framework for specifying and detecting antipatterns in SBSs. Using our approach, we specify ten well-known and common antipatterns, including Multi Service and Tiny Service, and automatically generate their detection algorithms. We apply and validate the detection algorithms in terms of precision and recall on two systems developed independently, (1) Home-Automation, an SBS with 13 services, and (2) FraSCAti, an open-source implementation of the Service Component Architecture (SCA) standard with more than 100 services. This validation demonstrates that our approach enables the specification and detection of SOA antipatterns with an average precision of $90 \%$ and recall of $97.5 \%$.
\end{abstract}

Keywords: Antipatterns; Service based systems; Service Component Architecture; Spec- 
ification; Detection; Quality of service; Design; Software evolution and maintenance.

\section{Introduction}

Service Oriented Architecture (SOA) is an emerging architectural style that is becoming broadly adopted in industry because it allows the development of low-cost, flexible, and scalable distributed systems by composing ready-made services, i.e., autonomous, reusable, and platform-independent software units that can be accessed through a network, such as the Internet [1]. This architectural style can be implemented utilizing a wide range of SOA technologies, such as OSGi, SCA, REST, RPC, and Web Services. SOA allows building different types of Service Based Systems (SBSs) from business systems to cloud-based systems. Google Maps, Amazon, eBay, PayPal, and FedEx are examples of SBSs. Spanoudakis and Mahbub defined SBSs as the composite systems, which are dynamically composed with autonomous Web services such that they are controlled by some composition processes [2]. We further generalise the definition:

"SBSs are systems that are built on top of SOA principles and are composed of services implemented with heterogeneous technologies as their building blocks."

However, the emergence of such systems raises several software engineering challenges. Indeed, like any other complex software systems, SBSs must evolve to fit new user requirements in terms of functionalities and Quality of Service (QoS). SBSs must also evolve to conciliate new execution contexts, such as addition of new devices, technologies, and-or protocols. All of these changes may degrade the design and QoS of SBSs and may often result in the appearance of common poor solutions to recurring problems, called Antipatterns - by opposition to design patterns, which are good solutions to problems that software engineers face when designing and developing systems. In addition to the degradation of the design and QoS, antipatterns resulting from these changes make it hard for software engineers to maintain and evolve systems.

Multi Service and Tiny Service are two common antipatterns in SBSs and it has been shown, in particular, that Tiny Service is the root cause of many SOA failures [3]. Multi Service is an SOA antipattern that represents a service implementing a multitude of methods related to different business and technical abstractions. Such a service is not easily reusable because of the low cohesion of its methods and is often unavailable to end-users because it is overloaded. Conversely, Tiny Service is a small service with just a few methods, which only implements part of an abstraction. Such service often requires several coupled services to be used together, leading to higher development complexity and reduced flexibility. These two antipatterns represent extreme in the balance between cohesion and coupling.

The automatic detection of such antipatterns is an important activity to assess the design and QoS of SBSs and ease the maintenance and evolution tasks of software engineers. However, few works have been devoted to SOA antipatterns and methods and techniques for the detection of antipatterns in SBSs are still in 
their infancy. In this paper, we consider the SCA systems to analyse and to detect antipatterns while keep other SOA technologies as our future work. However, the availability of free SBSs as test bed is a major challenge that we might face.

Our goal is to assess the design and QoS of SBSs. To achieve this goal, we already proposed a novel and innovative approach, SODA (Service Oriented Detection for Antipatterns) supported by a framework, SOFA (Service Oriented Framework for Antipatterns), to specify and detect SOA antipatterns automatically in SBSs [4]. This framework supports the static and dynamic analysis of SBSs, along with their combination. Static analysis involves measurement of structural properties related to the design of SBSs while dynamic analysis requires the runtime execution of SBSs for the measurement of runtime properties, mainly related to QoS.

The SODA approach relies on the first language to specify SOA antipatterns in terms of metrics. This language is defined from a thorough domain analysis of SOA antipatterns from the literature. It allows the specifications of SOA antipatterns using high-level domain-related abstractions. It also allows the adaptation of the specifications of antipatterns to the context of the analysed SBSs. Using this language and the SOFA framework dedicated to the static and dynamic analysis of SBSs, we generate detection algorithms automatically from the specifications of SOA antipatterns and apply them on any SBSs under analysis. The originality of our approach stems from the ability for software engineers to specify SOA antipatterns at a high-level of abstraction using a consistent vocabulary and from the use of a domain-specific language for automatically generating the detection algorithms.

In our previous work [4], we assessed the effectiveness of the proposed approach only on a small scale SBS, i.e., Home-Automation. The results indicated that we had good precision (92.5\%) and recall $(100 \%)$ on Home-Automation, after specifying SOA antipatterns and generating their detection algorithms automatically. In the extension to our previous work, we apply SODA by specifying 10 well-known and common SOA antipatterns and generating their detection algorithms. Then, we validate the detection results in terms of precision and recall on two systems: (1) Home-Automation, an SBS developed independently by two Masters students that involves 13 services and (2) FraSCAti [5,6], an open-source implementation of the SCA standard with more than 100 services. FraSCAti is almost ten times bigger than Home-Automation in terms of the number of constituent services. We also consider two different versions of Home-Automation: (a) an original version, which includes 13 services and (b) a version modified by adding and modifying services to inject intentionally some antipatterns. We show that SODA allows the specification and detection of a representative set of SOA antipatterns with an average precision of $90.84 \%$ and a recall of $97.5 \%$.

In summary, our contributions from [4] include:

(1) A first approach, SODA, for specifying and detecting SOA antipatterns supported by an underlying framework, SOFA; 
(2) An extensible domain specific language (DSL) to specify SOA antipatterns;

(3) A validation of the approach with a small scale SBS, i.e., Home-Automation.

This paper extends our previous work [4] with the following additional contribution: an extensive validation of the proposed approach with a large scale SBS, i.e., FraSCAti [5,6], which is an open-source implementation of the SCA standard, and the largest SCA system available. We also complement our previous work [4] with a more elaborated and up-to-date related work. In addition, we also specify three new SOA antipatterns and perform their detection on FraSCAti. Thus, we extend our domain specific language (DSL) to accommodate new metrics, and extend the repository of detected antipatterns.

The remainder of this paper is organised as follows. Section 2 surveys related work on the detection of antipatterns and patterns. Section 3 presents our specification and detection approach, SODA, along with the specification language and the underlying detection framework, SOFA. Section 4 presents experiments performed on Home-Automation and FraSCAti for validating our approach. Finally, Section 5 concludes and sketches future work.

\section{Related Work}

Architectural (or design) quality is essential for building well-designed, maintainable, and evolvable SBSs. Patterns and antipatterns have been recognized as one of the best ways to express architectural concerns and solutions. However, unlike Object Oriented (OO) antipatterns, methods and techniques for the detection and correction of SOA antipatterns are still in their infancy. The next few sections focus on works that deal with detecting SOA patterns, SOA antipatterns, and OO antipatterns.

\subsection{Detection of SOA Patterns}

The current catalog of SOA design patterns is rich enough: there exists a number of books on SOA patterns and principles [7-9] that provide guidelines and principles characterizing "good" service-oriented designs. Such books enable software engineers to manually evaluate the quality of their systems and provide a ground for improving design and implementation. For example, Rotem-Gal-Oz et al. [9] introduced 23 SOA patterns and four SOA antipatterns and discussed their consequences, causes, and corrections. Erl, in his book [7], introduced more than $80 \mathrm{SOA}$ design-, implementation-, security-, and governance-related patterns.

However, there are a very few contributions on detecting patterns in the SOA context. One prominent contribution towards detecting SOA patterns is by Upadhyaya et al. [10], in which the authors devised an approach based on execution log analysis. The authors detected 9 service-composition related SOA patterns that are mostly domain-specific and goal-oriented: if the application is related to the domain of financial or supply-chain management, then the composition patterns can eas- 
ily be observed based on the use-case scenarios. However, the proposed approach cannot assess the quality of an SBS design and its performance in general. There are also a few works on detecting SOA patterns at service-level, i.e., similarities among services [11], and at process-level, i.e., within workflows [12-14] but none of them considered patterns at design-level. Unlike these works, we are interested in patterns and antipatterns, that assess the quality of design and QoS to ease the maintenance and evolution of SBSs.

\subsection{Detection of SOA Antipatterns}

Unlike SOA patterns, fewer books and papers deal with SOA antipatterns: most references are Web sites where SOA practitioners share their experiences in SOA design and development [15-17]. In 2003, Dudney et al. [18] published the first book on SOA antipatterns. This book provides a catalog of 53 antipatterns related to the architecture, design, and implementation of systems based on J2EE technologies, such as EJB, JSP, Servlet, and Web Services. Most antipatterns described in this book cannot be detected automatically and are specific to a technology and correspond to variants of the Tiny and Multi Service. Král et al. [3] also described seven SOA antipatterns, which are caused by an improper usage of SOA standards and improper practices borrowed from the OO design style.

While the catalog of SOA antipatterns is growing, there are very few dedicated approaches for detecting SOA antipatterns in SBSs, i.e., the approach proposed by Trčka et al. [19] and ours in [4]. Trčka et al. [19] proposed a technique to discover data-flow antipatterns based on model-checking. They specified using temporal logic nine data-flow antipatterns (e.g., Missing Data, Inconsistent Data, Not Deleted on Time, etc.) and detected them by analysing data dependencies within workflows and improper data handling. In [4], we also proposed a means for the specification and detection of SOA antipatterns that we recall in the following for consistency. Trčka et al. focused on data-flow antipatterns whereas we focus on detecting antipatterns to assess and improve the quality of design and QoS of SBSs. However, we observe from the literature that due to the smaller 'catalog size' and limited available resources, i.e., articles, books, journals, etc., the specification and detection of SOA antipatterns were not considered with greater importance by the SOA community.

\subsection{Detection of OO Antipatterns}

In the contrary to the efforts given on detecting SOA patterns and antipatterns, a number of methods and tools exist for the detection of antipatterns in OO systems [20-25] and various books have been published on that topic. For example, Brown et al. [26] introduced a collection of 40 antipatterns, Beck, in Fowler's highly-acclaimed book on refactoring [27], compiled 22 code smells that are low-level antipatterns in source code, suggesting where engineers should apply refactorings. 
Among the previous works, DECOR [20] is a rule-based approach for the specification and detection of code and design smells in $\mathrm{OO}$ systems that can be related to our work. The authors use a domain specific language to specify smells and then automatically generate smell-detection algorithms, which are directly executable. DECOR can detect smells in OO systems with a precision of $60.5 \%$ and recall of 100\%. Later, Kessentini et al. [21] improved the detection precision by automating the rule construction. The authors use genetic programming for finding an optimal set of rules to maximize the smell detection. Also, Khomh et al. [28] proposed an GQM-based (Goal Question Metric) approach relying on the definition of antipattern rather than the rule card, and improved precision and recall from its state-of-the-art approach, DECOR. However, being inspired from DECOR, we intend to follow a similar approach in the context of the service-oriented paradigm for detecting SOA antipatterns.

Other related works have focused on the detection of specific antipatterns related to system's performance and resource usage and-or given technologies. For example, Wong et al. [29] used a genetic algorithm for detecting software faults and anomalous behavior related to the resource usage of a system (e.g., memory usage, processor usage, thread count). Their approach is based on utility functions, which correspond to predicates that identify suspicious behavior based on resource usage metrics. For example, a utility function may report an anomalous behavior corresponding to spam sending if it detects a large number of threads. In another relevant work, Parsons et al. [30] introduced an approach for the detection of performance antipatterns specifically in component-based enterprise systems (in particular, J2EE applications) using a rule-based approach relying on static and dynamic analyses.

Moreover, there exists tools proposed by the industry and the academic community for automating the detection of OO code smells and antipatterns, such as cbsdetector [31], FindBugs [32], iPlasma [33], JDeodorant [34], PatOMat [35], PMD [36], SonarQube [37], SPARSE [38], etc.

\subsection{Summary}

One of the root causes of $\mathrm{OO}$ antipatterns is the adoption of a procedural design style in OO system whereas for SOA antipatterns, it stems from the adoption of an OO style design in SOA system [3]. OO detection methods and tools cannot be directly applied to SOA because SOA focuses on services as first-class entities whereas OO focuses on classes, which are at a lower-level of granularity. Moreover, the highly dynamic nature of an SOA environment raises several challenges that are not faced in $\mathrm{OO}$ development and requires more dynamic analyses.

Although different, all these previous works on OO systems, SOA patterns, and performance antipattern detection form a sound basis of expertise and technical knowledge for building methods for the detection of SOA antipatterns. 


\section{The SODA Approach}

In [4], we proposed a three-step approach, named SODA, for the specification and detection of SOA antipatterns as shown in Figure 1. We recall this approach:

Step 1. Specify SOA antipatterns: This step lies in identifying properties in SBSs relevant to SOA antipatterns. These properties can also be referred to as metrics. Using those properties, we define a Domain-Specific Language (DSL) for specifying antipatterns at a high level of abstraction.

Step 2. Generate detection algorithms: In this step, detection algorithms are generated automatically from the specifications defined in the previous step.

Step 3. Detect automatically SOA antipatterns: The third step consists of applying, on the SBSs of interest, the detection algorithms generated in Step 2 to detect SOA antipatterns.

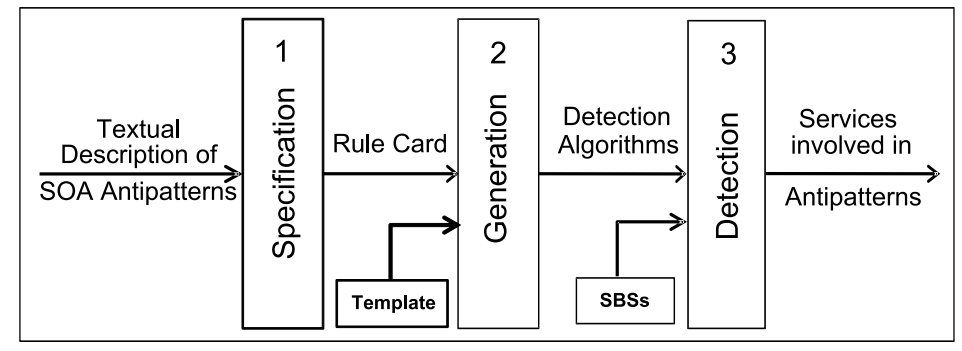

Figure 1. The SODA Approach.

The following sections describe the first two steps. The third step is described in Section 4, where we detail the experiments performed for validating SODA.

\subsection{Specification of SOA Antipatterns}

As a prerequisite to specify antipatterns, we perform a thorough domain analysis of SOA antipatterns by studying their definitions and specifications in the literature $[3,9,18]$ and in online resources and articles [15-17]. This domain analysis allows us to identify properties relevant to SOA antipatterns, including static properties related to their design (e.g., cohesion and coupling) and also dynamic properties, such as QoS criteria (e.g., response time and availability). Static properties are properties that apply to the static descriptions of SBSs, such as WSDL (Web Services Description Language, for Web Services) and SCDL (Service Component Definition Language, for SCA) files, whereas dynamic properties are related to the dynamic behavior of SBSs as observed during their execution. We use these properties as a base vocabulary to define a DSL, in the form of a rule-based language for specifying SOA antipatterns. The DSL offers software engineers with high-level domain-related abstractions and variability points to express different properties of antipatterns depending on their own judgment and context. 


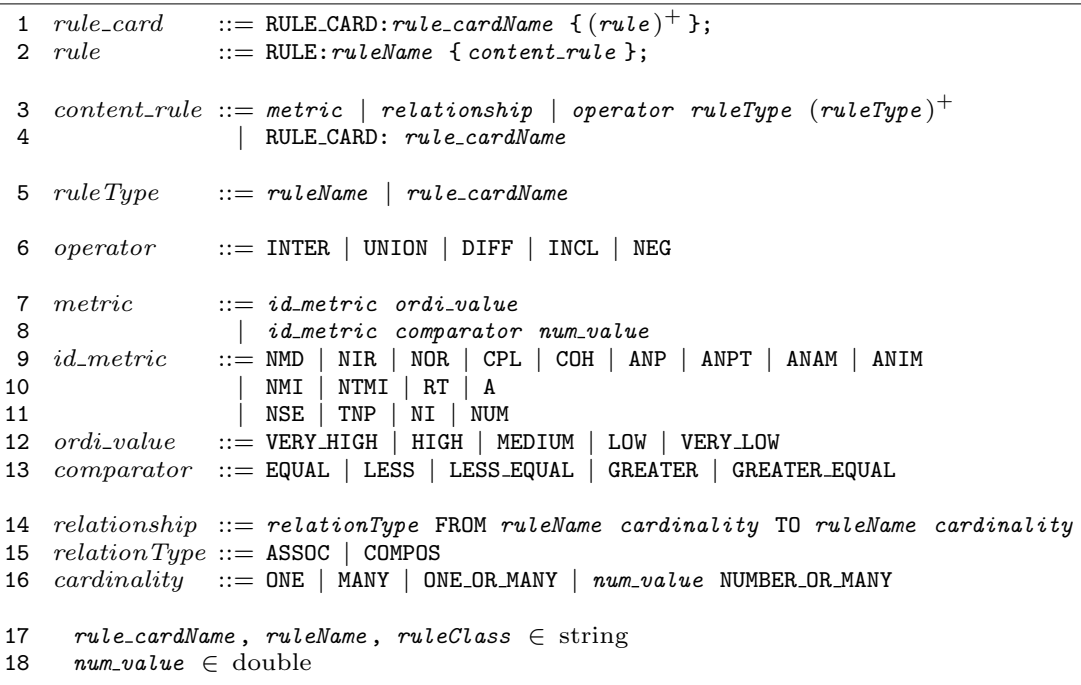

Figure 2. BNF Grammar of Rule Cards.

We specify antipatterns using rule cards, i.e., sets of rules. We formalize rule cards with a Backus-Naur Form (BNF) grammar, which determines the syntax of our DSL. Figure 2 shows an extended version of the grammar that we first proposed in [4] and used to express the rule cards. A rule card is identified by the keyword RULE_CARD, followed by a name and a set of rules specifying this specific antipattern (Figure 2, line 1). A rule (line 3 and 4) describes a metric, an association or composition relationship among rules (lines 14-16) or a combination with other rules, based on set operators including intersection, union, difference, inclusion, and negation (line 6). Each rule returns a set of services. Therefore, it is possible to apply a set operator between at least two rules and obtain the result of this set operation again in the form of a set of services. A rule can refer also to another rule card previously specified (line 4). A metric associates to an identifier a numerical or an ordinal value (lines 7 and 8). Ordinal values are defined with a five-point Likert scale: very high, high, medium, low, and very low (line 12). Numerical values are used to define thresholds with comparators (line 13), whereas ordinal values are used to define values compared to all the services of an SBS under analysis (line 12). We define ordinal values with the box-plot statistical technique [39] to relate ordinal values with concrete metric values while avoiding setting artificial thresholds.

The metric suite (lines 9-11) encompasses both static and dynamic metrics. The static metric suite includes (but is not limited to) the following metrics: number of methods declared (NMD), number of incoming references (NIR), number of outgoing references $(\mathrm{NOR})$, coupling $(\mathrm{CPL})$, cohesion $(\mathrm{COH})$, average number of parameters in methods (ANP), average number of primitive type parameters (ANPT), average num- 
ber of accessor methods (ANAM), and average number of identical methods (ANIM). The dynamic metric suite contains: number of method invocations (NMI), number of transitive methods invoked (NTMI), response time (RT), and availability (A).

The DSL shown in Figure 2 can be extended by adding new metrics and-or values. Later in Section 4.6 (see Table 6), we specify and detect three new SOA antipatterns, which require four new metrics. We also add these metrics to our DSL. The new metrics (line 11) include: number of services encapsulated (NSE), total number of parameters (TNP), number of interfaces (NI), and number of utility methods (NUM). Utility methods provide facilities with logging, data validation, andor notifications, rather than any business functionalities.

\subsubsection{Example of Rule Cards}

Figure 3 illustrates the grammar with the rule cards of the Multi Service and Tiny Service antipatterns. The Multi Service antipattern is characterized by very high response time and number of methods and low availability and cohesion. A Tiny Service corresponds to a service that declares a very low number of methods and has a high coupling with other services. For the sake of clarity, we illustrate the DSL with two intra-service antipatterns, i.e., antipatterns within a service. However, the DSL allows also the specification of inter-service antipatterns, i.e., antipatterns spreading over more than one service. We provide the rule cards of such other more complex antipatterns later in the experiments (see Section 4).

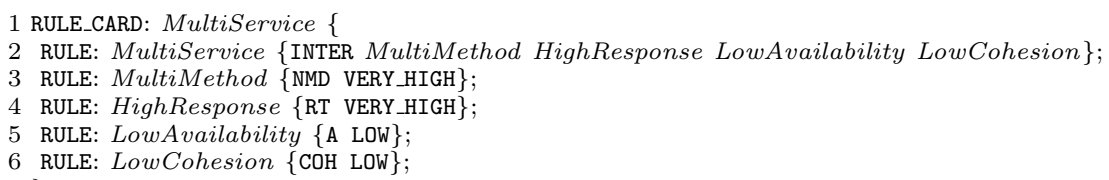

(a) Multi Service

1 RULE_CARD: TinyService \{

2 RULE: TinyService \{INTER FewMethod HighCoupling ;

3 RULE: FewMethod \{NMD VERY_LOW\};

4 RULE: HighCoupling \{CPL HIGH $\}$;

$5\}$

(b) Tiny Service

Figure 3. Rule Cards for Multi Service and Tiny Service.

Using a DSL offers greater flexibility than implementing ad hoc detection algorithms, because it allows describing antipatterns using high-level, domain-related abstractions and focusing on what to detect instead of how to detect it [40]. Indeed, the DSL is independent of any implementation concern, such as the computation of static and dynamic metrics and the multitude of SOA technologies underlying SBSs. Moreover, the DSL allows the adaptation of the antipattern specifications to 
the context and characteristics of the analysed SBSs by adjusting the metrics and associated values.

\subsection{Generation of Detection Algorithms}

From the specifications of the SOA antipatterns described with the DSL, we automatically generate the detection algorithms for all antipatterns like in [4]. In this paper, we improved the process of generation of detection algorithms. In [4], we used Kermeta [41] to automate the algorithm generation. However, in this paper, we used Ecore [42] and Acceleo [43] which are more recent meta-modeling frameworks than Kermeta.

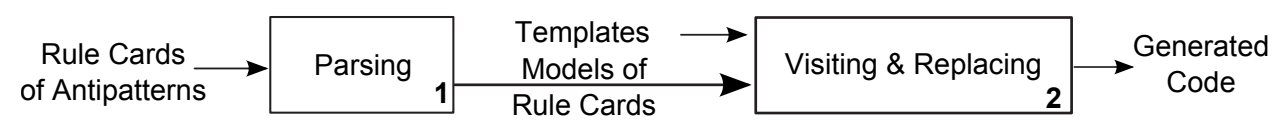

Figure 4. Generation of Detection Algorithms.

For the generation of the detection algorithms, first, we parse the rule cards of each antipattern and represent them as models. Then, we use Ecore to syntactically validate the rule card models against the meta-model of our DSL. Ecore also guarantees the correctness of the rule card models. We use a template-based code generation technique provided by Acceleo. To do this, we define a unique template for all rule cards consisting of well-defined tags to be replaced with the metric values defined in the rule cards of antipatterns. Finally, the template is applied to a rule card model resulting in a JAVA class, which is directly compilable and executable without any manual involvement.

Figure 5 shows the template for the Multi Service that we use to generate the detection code. In the first line of Figure 5 , the template includes the meta-model of our DSL. It also provides tags, which are identified in square brackets and correspond to the variables to be replaced with rule card name, rule names, metrics, values, and different operators. Only one template is required for all the rule cards. Thus, it is easy to maintain them.

Figure 6 shows the detection code generated by Acceleo based on the rule card and the template defined in Figure 5. This generation creates a JAVA class with the operators and different metrics with their concrete values. The class generated here is directly compilable and executable using the JAVA classloader. Engineers only need to provide the concrete implementation of the metrics referred by the rule card.

This generative process is fully automated to avoid any manual tasks, which are usually repetitive and error-prone. This process also ensures the traceability between the specifications of antipatterns with the DSL and their concrete detection in SBSs using our underlying framework. Consequently, software engineers can focus 


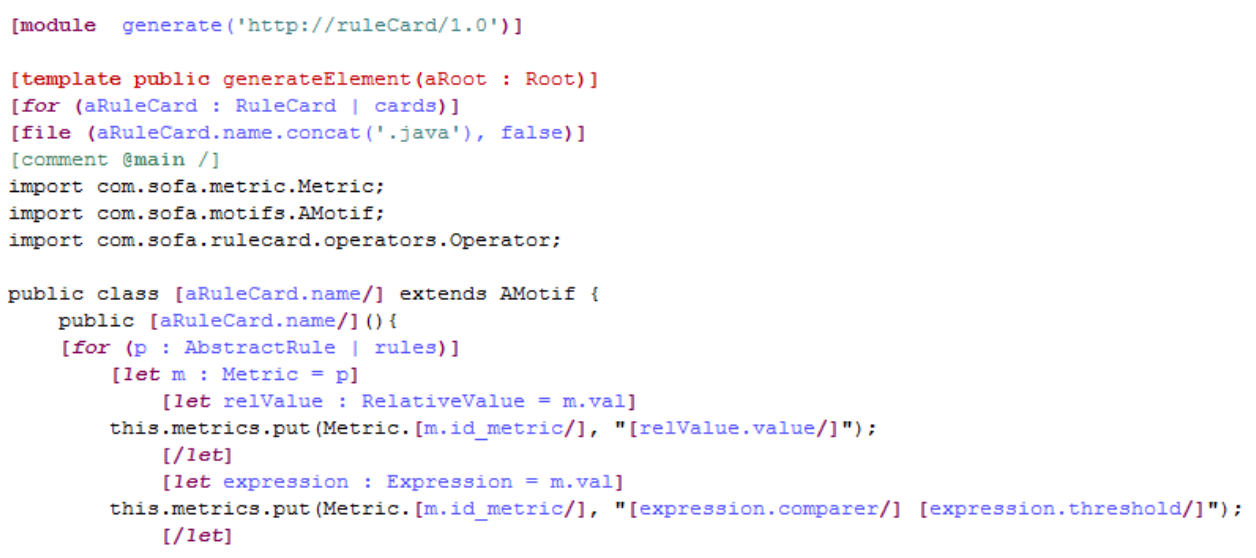

Figure 5. The Snapshot of the template for the Multi Service.

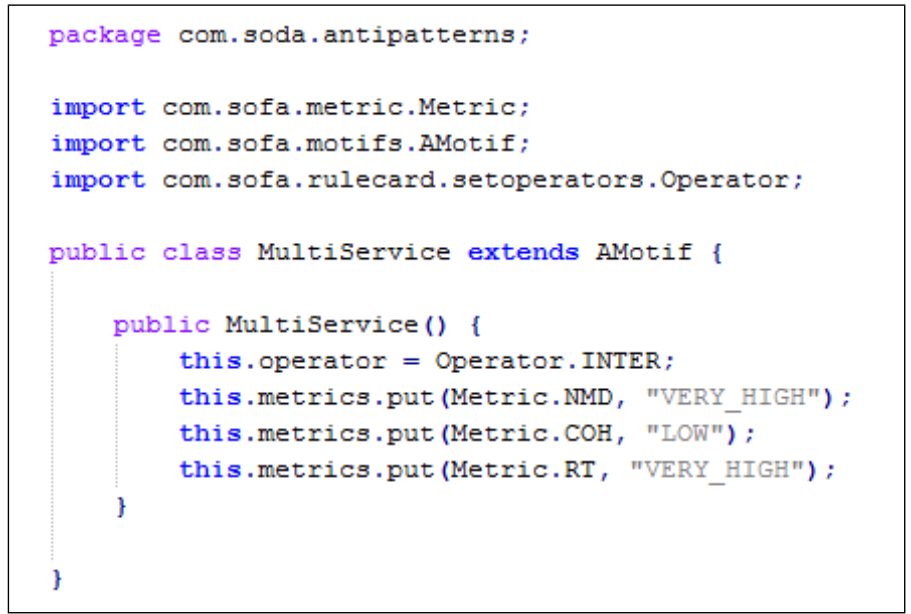

Figure 6. The Snapshot of the generated code for the Multi Service.

on the specification of antipatterns, without considering any technical aspects of the underlying framework.

\subsection{SOFA: Underlying Framework}

We develop a framework, called SOFA (Service Oriented Framework for Antipatterns), that supports the detection of SOA antipatterns in SBSs. This framework, designed itself as an SBS and illustrated in Figure 7, provides different services corresponding to the main steps for the detection of SOA antipatterns: (1) the automated generation of detection algorithms; (2) the computation of static and dynamic metrics; and (3) the specification of rules including different sub-services 
for the rule language, the box-plot statistical technique, and the set operators. The

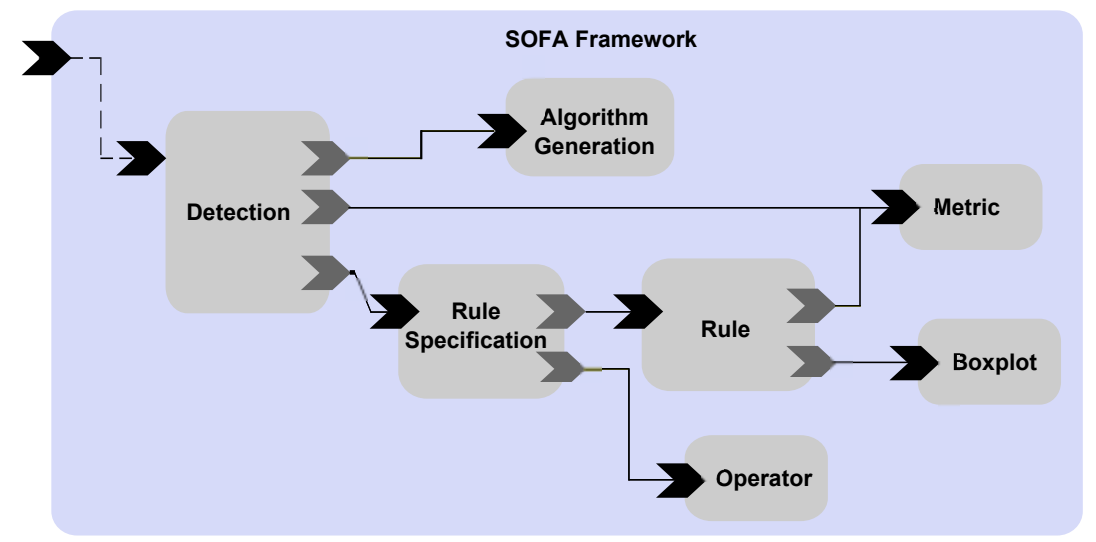

Figure 7. The SOFA Framework.

rule-specification and algorithm-generation services provide all constituents to describe models of rule cards as well as the algorithms to visit rule card models and to generate detection algorithms from these models. These different constituents rely on Model Driven Engineering techniques, which provide the means to define a DSL, parse it, and check its conformance with the grammar. We also use Ecore and Acceleo for generating the detection algorithms based on models of rule cards.

With respect to the computation of metrics, the generated detection algorithms call sensors and triggers implemented using the services provided by FraSCAti. These sensors and triggers, implemented as join points in an aspect-oriented programming style, allow, at runtime, the introspection of the interface of services and the triggering of events to add non-functional concerns, such as transactions, debugging, and, in our case, the computation of metrics such as response time.

These sensors and triggers are provided at the deployment of the SBS under analysis. The code excerpt shown in Figure 8 presents the computation of the response time as a join point at the service interface level. The sensor RTIntentHandler (line 1) corresponds to an aspect that will intercept a service call and monitor the service response time. An intent join point (line 2) corresponds to the interface where a service invocation has been intercepted. The code enabling the computation of the response time is inserted before and after the invocation of the service (line 5). This new monitoring aspect is then declared as a service and added to the SOFA framework within the metric module (line 7).

SCA is a relatively new standard, advocated by researchers and major software vendors, like IBM and Oracle, for developing technology-agnostic and extensible SBSs. FraSCAti encompasses different modules for binding, deploying, running, and monitoring SBSs. Such a platform, i.e., FraSCAti is essential for allowing the 
1: public class RTIntentHandler implements IntentHandler \{

2: public Object invoke(IntentJoinPoint ijp) \{

3: long startTime $=$ System.currentTimeMillis();

4: Object ret $=$ null;

5: ret $=$ ijp.proceed () ;

6: long estimatedTime $=$ System.currentTimeMillis () - startTime;

7: Metrics.setValue( “RT”,estimatedTime);

8: return ret;

9: $\}$

10: $\}$

Figure 8. The Example of the usage of Sensors and Triggers.

detection of SOA antipatterns at execution time [6], which provides also runtime support for the SCA standard. Furthermore, the SOFA framework is implemented itself as an SCA component to ease its use and evolution and to offer it as a service to end-users concerned by the design and QoS of their SBSs. Currently, a prototype version of SOFA is available on http://sofa.uqam.ca/soda.html.

\section{Experiments}

To demonstrate the completeness and extensibility of our DSL, the accuracy of the generated algorithms, and the usefulness of the detection results with their related performance, we performed experiments with 10 antipatterns on two different service-based SCA systems, i.e., Home-Automation and FraSCAti [5]. In our previous work [4], we presented detection results on Home-Automation only. In this paper, we perform similar experiments on FraSCAti while keeping the same assumptions as in [4].

\subsection{Assumptions}

The experiments aim at validating the following four assumptions:

A1. Generality: The DSL allows the specification of many different SOA antipatterns, from simple to more complex ones. This assumption supports the applicability of SODA using the rule cards on 10 SOA antipatterns, composed of 13 static and dynamic metrics.

A2. Accuracy: The generated detection algorithms have a recall of 100\%, i.e., all existing antipatterns are detected, and a precision greater than $75 \%$, i.e., more than three-quarters of detected antipatterns are true positive. Given the trade-off between precision and recall, we assume that $75 \%$ precision is significant enough with respect to $100 \%$ recall. This assumption supports the precision of the rule cards and the accuracy of the algorithm generation and of the SOFA framework. 
A3. Extensibility: The DSL and the SOFA framework are extensible for adding new SOA antipatterns. Through this assumption, we show how well the DSL, and in particular the metrics, with the supporting SOFA framework, can be combined to specify and detect new antipatterns.

A4. Performance: The computation time required for the detection of antipatterns using the generated algorithms is reasonably very low, i.e., in the order of few seconds. This assumption supports the performance of the services provided by the SOFA framework for the detection of antipatterns.

\subsection{Subjects}

We apply our SODA approach using the SOFA framework to specify 10 different SOA antipatterns. Table 1 summarizes these antipatterns that we specified in [4], of which the first seven are from the literature and three others has been defined in our previous work, namely, Bottleneck Service, Service Chain, and Data Service. These new antipatterns are inspired from OO code smells [27]. In these summaries, we highlight in bold the key concepts relevant for the specification of their rule cards given in Figure 9.

\subsection{Objects}

We perform the experiments on two different service-based SCA systems, i.e., HomeAutomation and FraSCAti [5,6]. Home-Automation has been developed independently for controlling remotely many basic household functions for elderly homecare support. It includes 13 services with a set of 7 predefined use-case scenarios for executing it at runtime. We use two versions of Home-Automation: the original version of the system, which includes 13 services, and a version modified by adding and modifying services to inject intentionally some antipatterns. The modifications on Home-Automation have been performed by an independent engineer to avoid biasing the results.

Figures 11 and 12 show the high-level design for Home-Automation ${ }^{\mathrm{a}}$ and FraS$C A t i^{\mathrm{b}}$ respectively. Among more than 100 services, Figure 12 shows only the principal service components of FraSCAti.

FraSCAti is itself implemented as an SCA system composed of 13 SCA composites, for a total of $91 \mathrm{SCA}$ components, with 130 provided services. FraSCAti is the largest SCA system currently freely available. It is an open source implementation of the SCA standard, and supports components implementation with different technologies (BPEL, Java, Spring, OSGi, and Java supported languages, etc.) and also multiple binding technologies for communication between components (i.e., RMI, SOAP, REST, JSON, JNA, and UPnP). Like Home-Automation, in FraSCAti we define a set of 6 scenarios and execute them to perform the detection.

\footnotetext{
${ }^{a}$ websvn.ow2.org/listing.php?repname $=$ frascati\&path $=/$ trunk/demo/home-automation $/$

$\mathrm{b}_{\text {frascati.ow2.org/doc/1.4/ch12s04.html }}$
} 


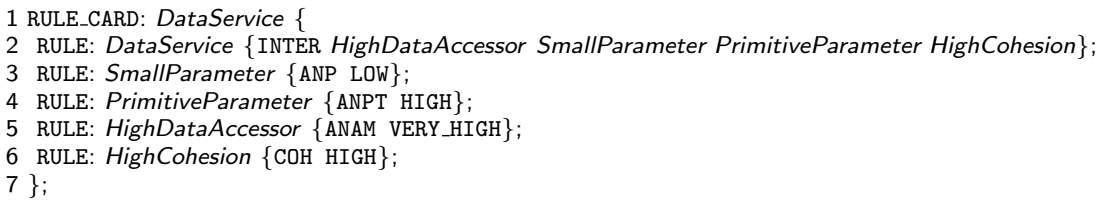

(a) Data Service

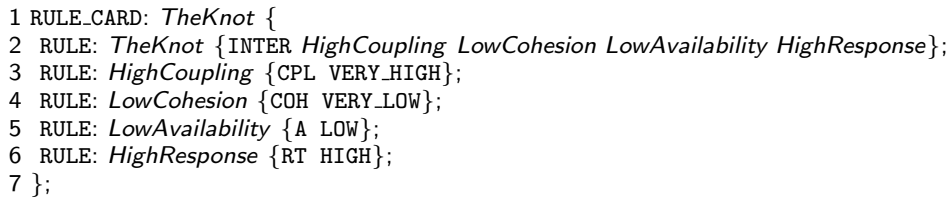

(b) The Knot

1 RULE_CARD: ChattyService \{

2 RULE: ChattyService $\{$ INTER Totallnvocation DSRuleCard $\}$;

3 RULE: DSRuleCard \{RULE_CARD: DataService\};

4 RULE: Totallnvocation \{NMI VERY_HIGH ;

$5\}$;

(c) Chatty Service

1 RULE_CARD: NobodyHome

2 RULE: NobodyHome \{INTER IncomingReference Methodlnvocation\};

3 RULE: IncomingReference \{NIR GREATER 0$\}$;

4 RULE: Methodlnvocation \{NMI EQUAL 0\};

$5\}$

(d) Nobody Home

1 RULE_CARD: BottleneckService \{

2 RULE: BottleneckService \{INTER LowPerformance HighCoupling \};

3 RULE: LowPerformance \{INTER LowAvailability HighResponse\};

4 RULE: HighResponse $\{\mathrm{RT} \mathrm{HIGH}\}$;

5 RULE: LowAvailability $\{$ A LOW $\}$;

6 RULE: HighCoupling \{CPL VERY_HIGH $\}$;

7 \};

(e) Bottleneck Service

1 RULE_CARD: SandPile \{

2 RULE: SandPile \{COMPOS FROM ParentService ONE TO ChildService MANY\};

3 RULE: ChildService \{ASSOC FROM ContainedService MANY TO DataSource ONE\};

4 RULE: ParentService $\{\mathrm{COH} \mathrm{HIGH}\}$;

5 RULE: DataSource \{RULE_CARD: DataService\}:

6 RULE: ContainedService $\{$ NRO > 1\};

7 \};

(f) Sand Pile

1 RULE_CARD: ServiceChain \{

2 RULE: ServiceChain \{INTER Transitivelnvocation LowAvailability\};

3 RULE: Transitivelnvocation \{NTMI VERY_HIGH ;

4 RULE: LowAvailability $\{$ A LOW $\}$

$5\}$

(g) Service Chain

1 RULE_CARD: DuplicatedService \{

2 RULE: DuplicatedService \{ANIM HIGH ;

$3\}$

(h) Duplicated Service

Figure 9. Rule Cards for Different Antipatterns. 
Table 1. List of Antipatterns. (The first seven antipatterns are extracted from the literature and three others are newly defined.)

Multi Service also known as God Object corresponds to a service that implements a multitude of methods related to different business and technical abstractions. This service aggregates too many methods into a single service, such a service is not easily reusable because of the low cohesion of its methods and is often unavailable to end-users because of it is overloaded, which may also induce a high response time [18].

Tiny Service is a small service with few methods, which only implements part of an abstraction. Such service often requires several coupled services to be used together, resulting in higher development complexity and reduced usability. In the extreme case, a Tiny Service will be limited to one method, resulting in many services that implement an overall set of requirements [18].

Sand Pile is also known as "Fine-Grained Services". It appears when a service is composed by multiple smaller services sharing common data. It thus has a high data cohesion. The common data shared may be located in a Data Service antipattern (see below) [3].

Chatty Service corresponds to a set of services that exchange a lot of small data of primitive types usually with a Data Service antipattern. The Chatty Service is also characterized by a high number of method invocations. Chatty Services chat a lot with each other [18].

The Knot is a set of very low cohesive services, which are tightly coupled. These services are thus less reusable. Due to this complex architecture, the availability of these services may be low, and their response time high $[9]$.

Nobody Home corresponds to a service, defined but actually never used by clients. Thus, the methods from this service are never invoked, even though it may be coupled to other services. Yet, it still requires deployment and management, despite of its non-usage [16].

Duplicated Service, a.k.a. The Silo Approach, introduced by IBM, corresponds to a set of highly similar services. Because services are implemented multiple times as a result of the silo approach, there may have common or identical methods with the same names and-or parameters [15].

Bottleneck Service is a service that is highly used by other services or clients. It has a high incoming and outgoing coupling. Its response time can be high because it may be used by too many external clients, for which clients may need to wait to get access to the service. Moreover, its availability may also be low due to the traffic

Service Chain a.k.a. Message Chain [27] in OO systems corresponds to a chain of services. The Service Chain appears when clients request consecutive service invocations to fulfill their goals. This kind of dependency chain reflects the subsequent invocation of services.

Data Service, a.k.a. Data Class [27] in OO systems, corresponds to a service that contains mainly accessor methods, i.e., getters and setters. In the distributed applications, there can be some services that may only perform some simple information retrieval or data access to such services. Data Services contain usually accessor methods with small parameters of primitive types. Such service has a high data cohesion.

Table 3 shows different structural properties for the two different versions of Home-Automation and FraSCAti. Details on the two versions of Home-Automation system including all the scenarios and involved services are available online at http://sofa.uqam.ca/soda. More details on FraSCAti design are also available on the FraSCAti Web site (http://frascati.ow2.org). 
Table 2. List of Three New Antipatterns.

God Component in SCA technology corresponds to a component that encapsulates a multitude of services. This component represents high responsibility enclosed by many methods with many different types of parameters to exchange. It may have a high coupling with the communicating services. Being at the component-level, God Component is at a higher level of abstraction than the Multi Service, which is at the service-level, and usually aggregates a set of services [18].

Bloated Service is an antipattern related to service implementation where services in SOA become 'blobs' with one large interface and lots of parameters. Bloated Service performs heterogeneous operations with low cohesion among them. It results in a system with less maintainability, testability, and reusability within other business processes. It requires the consumers to be aware of many details (i.e., parameters) to invoke or customize them [17].

Stovepipe Service is an antipattern with large number of private or protected methods that primarily focus on performing infrastructure and utility functions (i.e., logging, data validation, notifications, etc.) and few business processes (i.e., data type conversion), rather than focusing on main operational goals (i.e., very few public methods). This may result in services with duplicated code, longer development time, inconsistent functioning, and poor extensibility [18].

1 RULE_CARD: GodComponent

2 RULE: GodComponent \{INTER HighEncapsulatedService MultiMethod HighParameter\};

3 RULE: HighEncapsulatedService \{NOSE HIGH

4 RULE: MultiMethod \{NMD VERY_HIGH $\}$;

5 RULE: HighParameter $\{$ TNP VERY_HIGH $\}$;

$6\}$

(a) God Component

1 RULE_CARD: BloatedService \{

2 RULE: BloatedService \{INTER Singlelnterface MultiMethod HighParameter LowCohesion\};

3 RULE: Singlelnterface \{NOI EQUAL 1\};

4 RULE: MultiMethod \{NMD VERY_HIGH\};

5 RULE: HighParameter $\{$ TNP VERY_HIGH $\}$;

6 RULE: LowCohesion $\{\mathrm{COH}$ LOW $\}$;

7 \};

(b) Bloated Service

1 RULE_CARD: StovepipeService \{

2 RULE: StovepipeService \{INTER HighUtilMethod FewMethod DuplicatedCode\};

3 RULE: HighUtilMethod \{NUM VERY_HIGH ;

4 RULE: FewMethod \{NMD VERY LOW $\}$ :

5 RULE: DuplicatedCode $\{$ ANIM HIGH $\}$;

$6\}$

(c) Stovepipe Service

Figure 10. Rule cards for three new Antipatterns

\subsection{Process}

Using the SOFA framework, we generated the detection algorithms corresponding to the rule cards of the 10 antipatterns. Then, we applied these algorithms at runtime on the Home-Automation system using its set of 7 predefined scenarios and 


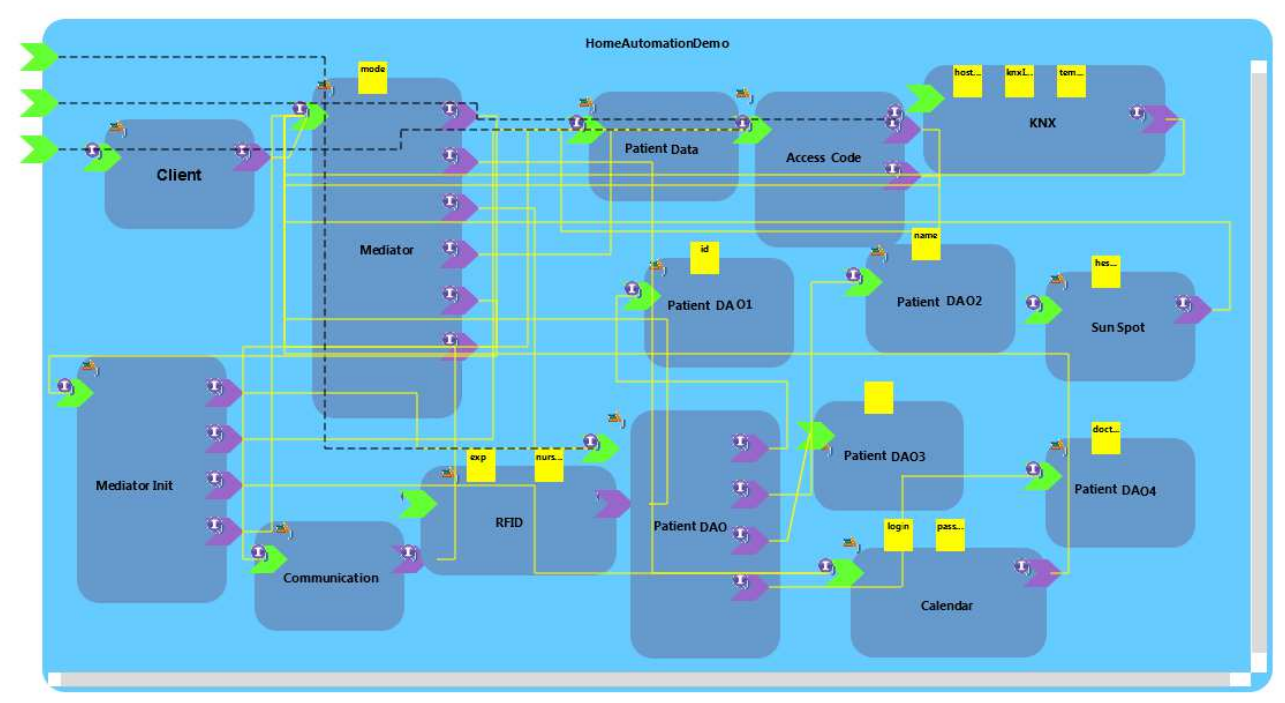

Figure 11. The Design of Home-Automation System.

Table 3. Structural properties for the two versions of Home-Automation and FraSCAti (NOS: Number of Services, NOM: Number of Methods, NOC: Number of Classes).

\begin{tabular}{|l|c|c|c|c|c|}
\hline System Name & Version & Size & NOS & NOM & NOC \\
\hline \hline Home Automation 1.0 & original & 3.2KLOC & 13 & 226 & 48 \\
Home Automation 1.1 & evolved & 3.4 KLOC & 16 & 243 & 52 \\
FraSCAti & original & 26.75KLOC & 130 & 1882 & 403 \\
\hline
\end{tabular}

on FraSCAti. Finally, we validated the detection results by analysing the suspicious services manually to: (1) validate that these suspicious services are true positives and (2) identify false negatives (if any), i.e., missing antipatterns. For this last validation step, we use the measures of precision (Equation 1), recall (Equation 2) and $\mathrm{F}_{1}$-measure (Equation 3) [44]. Precision estimates the ratio of true antipatterns identified among the detected antipatterns, while recall estimates the ratio of detected antipatterns among the existing antipatterns.

$$
\begin{gathered}
\text { precision }=\frac{\mid\{\text { existing antipatterns }\} \cap\{\text { detected antipatterns }\} \mid}{\mid\{\text { detected antipatterns }\} \mid} \\
\text { recall }=\frac{\mid\{\text { existing antipatterns }\} \cap\{\text { detected antipatterns }\} \mid}{\mid\{\text { existing antipatterns }\} \mid}
\end{gathered}
$$

We also compute $\mathrm{F}_{1}$-measure, i.e., the weighted average of precision and recall, to measure the accuracy of our detection algorithms. 


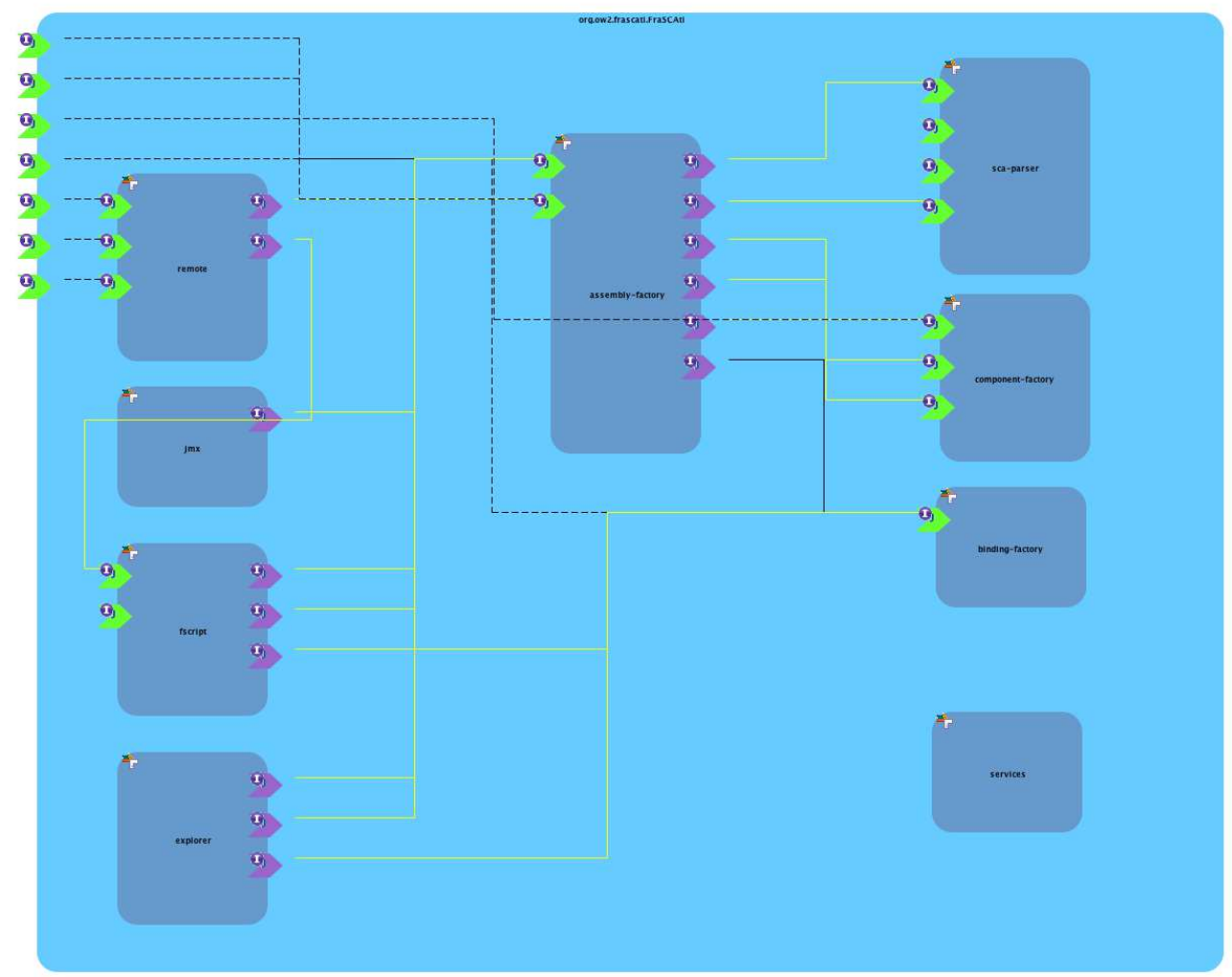

Figure 12. The Design of FraSCAti taken from FraSCAti Web site.

$$
F_{1} \text {-measure }=2 \times \frac{\text { precision } \times \text { recall }}{\text { precision }+ \text { recall }}
$$

The validation for Home-Automation and FraSCAti has been carried out manually by an independent software engineer. We provided him the complete descriptions of the target SOA antipatterns, and the complete design of the two versions of Home-Automation and FraSCAti. Manual validation is laborious task and may require 1 to 3 days for an engineer to validate depending on the size of the target SBS. We also reported the FraSCAti detection results to its development team. The FraSCAti development team provided their feedback stating if they agree or not with our preliminary detection results including the Multi Service, Tiny Service, Chatty Service, The Knot, Bottleneck Service, and Data Service. They validate our detection results based on the antipatterns textual descriptions and the rule cards we defined, and they agreed with us on five out of these six antipatterns. They do not validate the Multi Service because of the subjectivity of the rule card, that we modified afterwards. 


\subsection{Results}

We present here the detection results for Home-Automation and FraSCAti followed by a general discussion. We then discuss the assumptions for both the systems in Section 4.6.

\subsubsection{Results on Home-Automation}

Table 4 presents the results for the detection of the 10 SOA antipatterns on the original and evolved version of Home-Automation that we also presented in [4]. For each antipattern, the table reports the involved services in the second column, the analysis method: static (S) and-or dynamic (D) in the third, then the metric values of rule cards in the fourth, and finally the detection times in the fifth. The three last columns report the precision, recall, and $\mathrm{F}_{1}$-measure.

\subsubsection{Details of the Results on Home-Automation}

We briefly present the detection results of the Tiny Service and Multi Service. The service IMediator has been identified as a Multi Service because of its very high number of methods (i.e., NMD equal 13) and its low cohesion (i.e., COH equal 0.027). These metric values have been evaluated by the Boxplot service respectively as high and low in comparison with the metric values of other services of Home-Automation. For example, for the metric NMD, the Boxplot estimates the median value of NMD in Home-Automation as equal to 2. In the same way, the detected Tiny Service has a very low number of methods (i.e., NMD equal 1) and a high coupling (i.e., CPL equal 0.44 ) with respect to other values. The values of the cohesion $\mathrm{COH}$ and coupling CPL metrics range from 0 to 1 . In the original version of Home-Automation, we did not detect any Tiny Service. We then extracted one method from IMediator and moved it in a new service named MediatorDelegate and then this service has been detected as a Tiny Service.

We also detected seven other antipatterns within the original version of HomeAutomation (see Table 4), namely, Duplicated Service, Chatty Service, Sand Pile, The Knot, Bottleneck Service, Data Service, and Service Chain. All these antipatterns involve more than one service, except Data Service and Duplicated Service. The service PatientDAO has been detected as a Data Service because it performs simple data accesses. Moreover, in the evolved version, we detected the Nobody Home antipattern, after an independent developer introduced the service UselessService, which is defined but never used in any scenarios. We detected a consecutive chain of invocations of IMediator $\rightarrow$ SunSpotService $\rightarrow$ PatientDAO $\rightarrow$ PatientDA02, which forms a Service Chain, whereas engineers validated IMediator $\rightarrow$ PatientDAO $\rightarrow$ PatientDA02. Therefore, we had precision of $75 \%$ and recall of $100 \%$ for the Service Chain antipattern. Therefore, we had precision of $75 \%$ and recall of $100 \%$ for the Service Chain antipattern. The SunSpotService was not validated by the engineers and thus, it was considered as a false positive. However, 
Table 4. Results for the detection of 10 SOA Antipatterns in the original and evolved version of Home-Automation system (S: Static, D: Dynamic).

\begin{tabular}{|c|c|c|c|c|c|c|c|}
\hline AntipatternName & ServicesInvolved & Analysis & Metrics & Time & Precision & Recall & $\mathrm{F}_{1}$ \\
\hline Tiny Service & MediatorDelegate & $\mathrm{S}$ & $\begin{array}{l}\text { NOR }=4 \\
\text { CPL }=0.440 \\
\text { NMD }=1\end{array}$ & $0.194 \mathrm{~s}$ & $\begin{array}{l}{[1 / 1]} \\
100 \%\end{array}$ & $\begin{array}{l}{[1 / 1]} \\
100 \%\end{array}$ & $100 \%$ \\
\hline Multi Service & IMediator & $S, D$ & $\begin{array}{l}\mathrm{COH}=0.027 \\
\mathrm{NMD}=13 \\
\mathrm{RT}=132 \mathrm{~ms}\end{array}$ & $0.462 \mathrm{~s}$ & $\begin{array}{l}{[1 / 1]} \\
100 \%\end{array}$ & $\begin{array}{l}{[1 / 1]} \\
100 \%\end{array}$ & $100 \%$ \\
\hline Duplicated Service & $\begin{array}{l}\text { CommunicationService } \\
\text { IMediator }\end{array}$ & $\mathrm{S}$ & ANIM $=25 \%$ & $0.215 \mathrm{~s}$ & $\begin{array}{l}{[2 / 2]} \\
100 \%\end{array}$ & $\begin{array}{l}{[2 / 2]} \\
100 \%\end{array}$ & $100 \%$ \\
\hline Chatty Service & $\begin{array}{l}\text { IMediator } \\
------- \\
\text { PatientDAO }\end{array}$ & $S, D$ & $\begin{array}{l}\text { ANP }=1.0 ; \text { ANPT }=1.0 ; \\
\text { NMI }=3 ; \text { ANAM }=100 \% ; \\
\mathrm{COH}=0.167 \\
\text { ANPP }=1 . \overline{0} ; \overline{\mathrm{ANPT}}=1 . \overline{0} ; \\
\mathrm{NMI}=3 ; \mathrm{ANAM}=100 \% ; \\
\mathrm{COH}=0.167\end{array}$ & $0.383 \mathrm{~s}$ & $\begin{array}{l}{[2 / 2]} \\
100 \%\end{array}$ & $\begin{array}{l}{[2 / 2]} \\
100 \%\end{array}$ & $100 \%$ \\
\hline Nobody Home & UselessService & $S, D$ & $\begin{array}{ll}N I R>0 \\
N M I=0\end{array}$ & $1.154 \mathrm{~s}$ & $\begin{array}{l}{[1 / 1]} \\
100 \%\end{array}$ & $\begin{array}{l}1 / 1] \\
100 \%\end{array}$ & $100 \%$ \\
\hline Sand Pile & HomeAutomation & $\mathrm{S}$ & $\begin{array}{l}\text { NCS }=13 \\
\text { ANP }=1.0 \\
\text { ANPT }=1.0 \\
\text { ANAM }=100 \% \\
\mathrm{COH}=0.167\end{array}$ & $0.310 \mathrm{~s}$ & $\begin{array}{l}{[1 / 1]} \\
100 \%\end{array}$ & $\begin{array}{l}{[1 / 1]} \\
100 \%\end{array}$ & $100 \%$ \\
\hline The Knot & $\begin{array}{l}\text { IMediator } \\
\text { PatientDAO }\end{array}$ & $S, D$ & $\begin{array}{l}\mathrm{COH}=0.027 \\
\mathrm{NIR}=7 \\
\mathrm{NOR}=7 \\
\mathrm{CPL}=1.0 \\
\mathrm{RT}=57 \mathrm{~ms}\end{array}$ & $0.412 \mathrm{~s}$ & $\begin{array}{l}{[1 / 2]} \\
50 \%\end{array}$ & $\begin{array}{l}{[1 / 1]} \\
100 \%\end{array}$ & $66.67 \%$ \\
\hline Bottleneck Service & $\begin{array}{l}\text { IMediator } \\
------- \\
\text { PatientDAO }\end{array}$ & S, D & $\begin{array}{l}\text { NIR }=7 ; \mathrm{NOR}=7 ; \\
\mathrm{CPL}=1.0 ; \mathrm{RT}=40 \mathrm{~ms} \\
\mathrm{~N} \overline{\mathrm{IR}}=4 ; \overline{\mathrm{N}} \mathrm{O} \overline{\mathrm{R}}=\overline{4} ; \\
\mathrm{CPL}=0.57 ; \mathrm{RT}=2 \mathrm{~ms}\end{array}$ & $0.246 \mathrm{~s}$ & $\begin{array}{l}{[2 / 2]} \\
100 \%\end{array}$ & $\begin{array}{l}{[2 / 2]} \\
100 \%\end{array}$ & $100 \%$ \\
\hline Data Service & PatientDAO & $\mathrm{S}$ & $\begin{array}{l}\text { ANAM }=100 \% \\
\text { COH }=0.167 \\
\text { ANPT }=1.0 \\
\text { ANP }=1.0\end{array}$ & $0.268 \mathrm{~s}$ & $\begin{array}{l}{[1 / 1]} \\
100 \%\end{array}$ & $\begin{array}{l}{[1 / 1]} \\
100 \%\end{array}$ & $100 \%$ \\
\hline Service Chain & $\begin{array}{l}\text { IMediator } \\
\text { SunSpotService } \\
\text { PatientDA0 } \\
\text { PatientDA02 }\end{array}$ & D & NTMI $=4$ & $0.229 \mathrm{~s}$ & $\begin{array}{l}{[3 / 4]} \\
75 \%\end{array}$ & $\begin{array}{l}{[3 / 3]} \\
100 \%\end{array}$ & $85.71 \%$ \\
\hline \multicolumn{4}{|l|}{ AVERAGE } & $0.387 \mathrm{~s}$ & $\begin{array}{c}{[15 / 17]} \\
92.5 \%\end{array}$ & $\begin{array}{c}{[15 / 15]} \\
100 \%\end{array}$ & $95.24 \%$ \\
\hline
\end{tabular}

the detected chain exists in the system but only in one scenario. Engineers did not consider the full chain as harmful and therefore did not classify it as an antipattern. Also, our detection algorithm reported PatientDAO service as the Knot antipattern because the availability (A) value was discarded, which was very high, i.e., $100 \%$. In contrast, we specified the availability (A) in the Knot antipattern as low. Moreover, we detected the HomeAutomation itself as Sand Pile.

\subsubsection{Results on FraSCAti}

We perform another experiment with FraSCAti as a new contribution with respect to Home-Automation from [4]. Table 5 presents the results for the detection of the 10 SOA antipatterns in FraSCAti. For each antipattern, the table also reports the 
suspicious services in the second column, the metric values of rule cards in the third, and finally the detection times in the fourth. The three last columns report the precision, recall, and $\mathrm{F}_{1}$-measure.

Table 5. Results for the detection of 10 SOA Antipatterns in the original version of FraSCAti (S: Static, D: Dynamic).

\begin{tabular}{|c|c|c|c|c|c|c|c|}
\hline AntipatternName & ServicesInvolved & Analysis & Metrics & Time & Precision & Recall & $\mathrm{F}_{1}$ \\
\hline Tiny Service & sca-parser & $\mathrm{S}$ & $\mathrm{NMD}=1 ; \mathrm{CPL}=0.56$ & $0.067 \mathrm{~s}$ & $\begin{array}{l}{[1 / 1]} \\
100 \%\end{array}$ & $\begin{array}{l}{[1 / 1]} \\
100 \%\end{array}$ & $100 \%$ \\
\hline Multi Service & $\begin{array}{l}\text { juliac } \\
\text { Explörer-GUi - - }\end{array}$ & S, D & $\begin{array}{c}\mathrm{COH}=0.1 ; \mathrm{NMD}=5 ; \mathrm{RT}=1018 \mathrm{~ms} \\
\mathrm{n} / \mathrm{a}\end{array}$ & $0.066 \mathrm{~s}$ & $\begin{array}{l}{[1 / 1]} \\
100 \%\end{array}$ & $\begin{array}{l}{[1 / 2]} \\
50 \% \\
\end{array}$ & $66.67 \%$ \\
\hline Duplicated Service & not present & $\mathrm{S}$ & $\mathrm{n} / \mathrm{a}$ & $0.302 \mathrm{~s}$ & $\begin{array}{l}{[0 / 0]} \\
100 \%\end{array}$ & $\begin{array}{l}{[0 / 0]} \\
100 \%\end{array}$ & $100 \%$ \\
\hline Chatty Service & not present & $S, D$ & $\mathrm{n} / \mathrm{a}$ & $0.057 \mathrm{~s}$ & $\begin{array}{l}{[0 / 0]} \\
100 \%\end{array}$ & $\begin{array}{l}{[0 / 0]} \\
100 \%\end{array}$ & $100 \%$ \\
\hline Nobody Home & 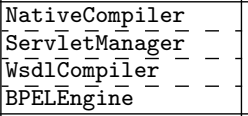 & $S, D$ & 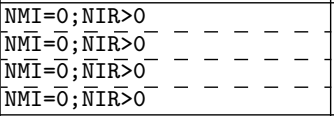 & $0.057 \mathrm{~s}$ & $\begin{array}{l}{[3 / 4]} \\
75 \%\end{array}$ & $\begin{array}{l}{[3 / 3]} \\
100 \%\end{array}$ & $85.71 \%$ \\
\hline Sand Pile & not present & $\mathrm{S}$ & $x_{1}$ & $0.058 \mathrm{~s}$ & $\begin{array}{l}{[0 / 0]} \\
100 \%\end{array}$ & $\begin{array}{l}{[0 / 0]} \\
100 \%\end{array}$ & $100 \%$ \\
\hline The Knot & sca-parser & $S, D$ & $\mathrm{CPL}=0.84 ; \mathrm{COH}=0.08 ; \mathrm{RT}=44 \mathrm{~ms}$ & $0.07 \mathrm{~s}$ & $\begin{array}{l}{[1 / 1]} \\
100 \% \\
\end{array}$ & $\begin{array}{l}{[1 / 1]} \\
100 \% \\
\end{array}$ & $100 \%$ \\
\hline Bottleneck Service & $\begin{array}{l}\text { sca-composite } \\
----- \\
\text { sca-parser }\end{array}$ & $S, D$ & $\begin{array}{l}\mathrm{RT}=41 \mathrm{~ms} ; \mathrm{CPL}=0.96 ; \\
\mathrm{NIR}=16 ; \mathrm{NOR}=8 \\
\mathrm{RT}=4 \overline{5} \overline{\mathrm{m}} ; \overline{\mathrm{CP}} \mathrm{L}=0 . \overline{84} ;--- \\
\mathrm{NIR}=16 ; \mathrm{NOR}=5\end{array}$ & $0.086 \mathrm{~s}$ & $\begin{array}{l}{[1 / 2]} \\
50 \%\end{array}$ & $\begin{array}{l}{[1 / 1]} \\
100 \%\end{array}$ & $66.67 \%$ \\
\hline Data Service & not present & $\mathrm{S}$ & $\mathrm{n} / \mathrm{a}$ & $0.07 \mathrm{~s}$ & $\begin{array}{l}{[0 / 0]} \\
100 \%\end{array}$ & $\begin{array}{l}{[0 / 0]} \\
100 \%\end{array}$ & $100 \%$ \\
\hline Service Chain & $\begin{array}{l}\text { MembraneGeneration } \\
\text { Processor } \\
\text { ComponentFactory } \\
\text { MembraneGeneration } \\
\text { TypeFactory } \\
\text { Processor } \\
\text { ComponentFactory } \\
\text { MembraneGeneration } \\
\text { Processor - } \\
\text { Processor } \\
\text { BindingFactory } \\
\text { PluginResolver }\end{array}$ & - & $\begin{array}{l}\text { NTMI }=4 \\
--------- \\
\text { NTMI }=4 \\
-------- \\
\text { NTMI }=4\end{array}$ & $0.056 \mathrm{~s}$ & $\begin{array}{c}{[2 / 3]} \\
66.67 \%\end{array}$ & $\begin{array}{l}{[2 / 2]} \\
100 \%\end{array}$ & $80 \%$ \\
\hline \multicolumn{4}{|l|}{ AVERAGE } & $0.089 \mathrm{~s}$ & $89.17 \%$ & $95 \%$ & $89.91 \%$ \\
\hline
\end{tabular}

\subsubsection{Details of the Results on FraSCAti}

We discuss some interesting detection results of FraSCAti reported in Table 5. We detect juliac as Multi Service because of its very high response time (RT equal $1,018 \mathrm{~ms}$ ), low cohesion ( $\mathrm{COH}$ equal 0.1 ), and high number of methods declared (NMD equal 5). The median values estimated by the Boxplot service, i.e., median of RT is $4 \mathrm{~ms}, \mathrm{COH}$ is 0.1 , and NMD is 1 compared to other services in FraSCAti classify juliac as Multi Service. juliac is likely a true positive because it implements six different features belonging to two different abstractions. Indeed, juliac provides services for the membrane generation and the Java compilers. Therefore, it is low cohesive 
and highly used because each component needs a membrane, and each composite file needs to be compiled by the Java compiler. Moreover, these actions are resourceconsuming and requires more execution time than other services. Manual inspection by the engineer also validated this detection.

Subsequently, the inspection of FraSCAti also allowed the identification of Explorer-GUI as a Multi Service. The FraSCAti development team confirmed that this service uses a high number of other services provided by FraSCAti. Indeed, this component encapsulates the graphical interface of FraSCAti Explorer, which aims to provide an exhaustive interface of FraSCAti functionalities. SOFA was not able to detect it because the execution scenarios did not involve the graphical interface of FraSCAti Explorer. Therefore, with one service detected as true positive, and with one missing occurrence of Multi Service, i.e., Explorer-GUI, we have a precision of $100 \%$ and recall of $50 \%$.

We also detect sca-parser as the Tiny Service with its small number of methods (NMD equal 1) and a high coupling (CPL equal 0.56). The engineer and the FraSCAti team also validated this detection. The boxplot median values are respectively 1 and 0.11 for NMD and CPL. After the manual inspection of FraSCAti implementation, the independent engineer identified that sca-parser contains only one method, i.e., parse(QName qname, ParsingContext parsingContext). In some cases, for a given metric, the median value and the high and-or low value might be identical if the values of most services are equal. For example, the median and low values are the same for NMD because out of 86 analysed services, 50 services have the NMD value of 1 . While concerned about the coupling CPL, sca-parser has dependency references to five other services (i.e., sca-metamodel-*) that give a high coupling. In fact, the coupling CPL values presented here are calculated on the logarithmic scale, i.e., the more references a service has to other services, the more highly coupled it is. The FraSCAti development team also validated sca-parser as a Tiny Service. However, according to them, sca-parser is invoked alone when only a reading for an SCA composite file is requested. However, FraSCAti performs more tasks than just reading and-or parsing an SCA composite file, and these other tasks are performed by other services such as AssemblyFactory. These several delegation also explains the high outgoing coupling. The appropriate detection of only one service as a Tiny Service leads to a precision and recall of $100 \%$.

The sca-composite and sca-parser services are detected as Bottleneck Service in FraSCAti with a high response time (i.e., RT values are $41 \mathrm{~ms}$ and $45 \mathrm{~ms}$, respectively) and a very high coupling (i.e., CPL values are 0.96 and 0.84 ). The coupling CPL is calculated by means of NIR and NOR, which are also very high for these two services. As estimated by the Boxplot service, the median for CPL and RT are 0.68 and $5 \mathrm{~ms}$ respectively. After manually analysing the FraSCAti design, the engineer found that only the sca-parser service is highly used by other services, and validated sca-parser as the only Bottleneck Service. The FraSCAti development team also agreed with this manual detection result, and confirmed that if too many external clients try to invoke the sca-parser service, they might wait to get the access. 
The precision and recall for Bottleneck Service are $50 \%$ and $100 \%$ respectively, with one false positive. The sca-composite is detected as Bottleneck Service and is a false positive again because of the value of availability (A), which is always very high, i.e., 100\%. For an SBS with several Composites, while the FraSCAti invokes the sca-parser service to parse all the relevant Composites, the sca-parser service naturally has the low availability. This low availability is mainly due to multiple invocations simultaneously by several clients to parse their Composites using the same sca-parser. Since, we have the availability (A) of $100 \%$ for all the components and did not consider the value of availability while reporting suspicious services, the sca-parser was reported as Bottleneck Service.

We also report three other detected SOA antipatterns in FraSCAti, namely \{NativeCompiler, ServletManager, WsdlCompiler, and BPELEngine $\}$ as Nobody Home, sca-parser as The Knot, and MembraneGeneration, TypeFactory, and Processor $\}$ as involved in a Service Chain. The BPELEngine has been detected as a Nobody Home antipattern because its implementation does not support the weaving of non-functional code such as sensors and triggers (see section 3.3). In fact, all the BPEL implementations of FraSCAti are, by default, discarded from the weaving algorithms provided by FraSCAti. We also detected one false positive for the Service Chain (Processor $\rightarrow$ Processor $\rightarrow$ BindingFactory $\rightarrow$ PluginResolver) that was not confirmed by the engineers. This detection was due to the fact that the Processor service calls itself using a public method and artificially extends the chain of calls. We may consider the modification of the Service Chain detection algorithm in order to eliminate self calls. Our detection algorithms do not detect any Duplicated Service, Chatty Service, Sand Pile, and Data Service antipatterns in FraSCAti. The absence of Sand Pile and Chatty Service is obvious, as they are related to Data Service and evidently, there is no such antipattern in FraSCAti, i.e., as confirmed by the FraSCAti team and our detection results. Our detection algorithms do not identify any suspicious services, after calculating the metric values we define in rule cards for those antipatterns. Indeed, as validated by FraSCAti team, there are no Duplicated Service and Data Service antipatterns in FraSCAti, and our detection algorithms do not filter any services as false positives either. Therefore, we obtain a precision and recall of $100 \%$. However, like in Home-Automation, the availability (A) for the services in FraSCAti is 100\%, because services are deployed locally.

In summary, very few services, i.e., 10 are actually involved in 6 antipatterns (4 antipatterns are not present) in FraSCAti, in comparison to the high number of services, i.e., 130 services. FraSCAti is well designed with continuous maintenance and evolution. Mostly, services (e.g., sca-parser and sca-composite-*) related to parsing and handling the composite file are involved in the antipatterns. The presence of such antipatterns in a system is not surprising because there is no other way to develop a parser without introducing a high coupling among services. More detailed on FraSCAti results are available at http://sofa.uqam.ca/soda.html. 


\subsection{Discussion on the Assumptions}

We now verify each of the four assumptions stated previously using the detection results.

A1. Generality: The DSL allows the specification of many different SOA antipatterns, from simple to more complex ones. Using our DSL, we specified 10 SOA antipatterns described in Table 1, as shown in rule cards given in Figure 3 and 9. These antipatterns range from simple ones, such as the Tiny Service and Multi Service, to more complex ones such as the Bottleneck and Sand Pile, which involve several services and complex relationships. In particular, Sand Pile has both the ASSOC and COMPOS relation types. Also, both Sand Pile and Chatty Service refer in their specifications to another antipattern, namely DataService. Thus, we show that we can specify from simple to complex antipatterns, which support the generality of our DSL.

A2. Accuracy: The generated detection algorithms have a recall of 100\%, i.e., all existing antipatterns are detected, and a precision greater than $75 \%$, i.e., more than three-quarters of detected antipatterns are true positive. For Home-Automation, as indicated in Table 4, we obtain a recall of $100 \%$, which means all existing antipatterns are detected, whereas the precision is $92.5 \%$. We have high precision and recall because the analysed system, Home-Automation is a small SBS with 13 services. Also, the evolved version includes two new services. Therefore, considering the small but significant number of services and the well defined rule cards using DSL, we obtain such a high precision and recall. For the original Home-Automation version, out of 13 services, we detected 6 services that are responsible for 8 antipatterns. Besides, we detected 2 services (out of 15 ) that are responsible for 2 other antipatterns in the evolved system.

Furthermore, for FraSCAti, as shown in Table 5, we achieve a recall of $95 \%$ and a precision of $89.17 \%$. The accuracy of our detection algorithms is acceptable, considering the high of number of services in FraSCAti, i.e., 130 services (see Table 3). According to our detection results, in total 15 services are responsible for 10 antipatterns in FraSCAti. We achieve our expected accuracy for detecting antipatterns even for a large SBS like FraSCAti and can support the second assumption.

A3. Extensibility: The DSL and the SOFA framework are extensible for adding new SOA antipatterns. The DSL has been initially designed for specifying the seven antipatterns described in the literature (see Table 1). Then, through inspection of the SBS and inspiration from OO smells, we added three new antipatterns, namely the Bottleneck Service, Service Chain, and Data Service. When specifying these new antipatterns, we reused four already-defined metrics and we added in the DSL and SOFA four more metrics (ANAM, NTMI, ANP, and ANPT). The language is flexible in the integration of new metrics. However, the underlying SOFA framework should also be extended to provide the operational implementations of the new metrics.

To further show the extensibility of our DSL and our framework, apart from 
Table 6. List of new metrics added to Our DSL

\begin{tabular}{|l|l|}
\hline Metric ID & \multicolumn{1}{|c|}{ Description } \\
\hline NSE & Number of Services Encapsulated \\
TNP & Total Number of Parameters \\
NI & Number of Interfaces \\
NUM & Number of Utility Methods \\
\hline
\end{tabular}

the antipatterns in [4], we also specify three more new SOA antipatterns in this paper, namely God Component, Bloated Service, and Stovepipe Service. Table 2 summarizes these three newly specified antipatterns and Figure 10 provides the corresponding rule cards. We defined four new metrics for specifying these new antipatterns. Table 6 lists these new metrics. The results of their detection on FraSCAti is reported in Table 7 . We report the suspicious services in column 2, and corresponding metric values for each new antipattern in column 4 . We also compute and report the precision, recall and $\mathrm{F}_{1}$-measure for each antipattern (columns 68). Such an addition can only be realized by skilled developers with our framework, which may require from 1 hour to 2 days according to the complexity of the metrics.

Thus, by extending the DSL with these three new antipatterns and integrating them within the SOFA framework for the detection, we support A3.

Table 7. Results for the detection of 3 new SOA Antipatterns in the original version of FraSCAti (S: Static)

\begin{tabular}{|c|c|c|c|c|c|c|c|}
\hline Antipattern & ServicesInvolved & Analysis & Metrics & Time & Precision & Recall & $\mathrm{F}_{1}$ \\
\hline God Component & \begin{tabular}{|l} 
FraSCAti \\
component-factory -
\end{tabular} & $S$ & $\begin{array}{l}\text { NOSE }=6 ; \mathrm{NMD}=12 ; \mathrm{TNP}=12 \\
\text { NOSE }=5 ; \mathrm{NMD}=7 ; \overline{T N P}=12\end{array}$ & $0.069 \mathrm{~s}$ & $\begin{array}{l}{[2 / 2]} \\
100 \% \\
\end{array}$ & $\begin{array}{l}{[2 / 2]} \\
100 \% \\
\end{array}$ & $100 \%$ \\
\hline Bloated Service & $\begin{array}{l}\text { component-factory } \\
---------- \\
\text { factory } \\
--------- \\
\text { frascati-binding-http }\end{array}$ & $S$ & 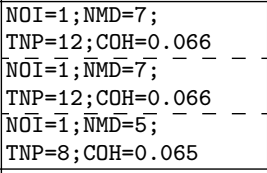 & $0.071 \mathrm{~s}$ & {$[3 / 3]$} & $\begin{array}{l}{[3 / 3]} \\
100 \%\end{array}$ & $100 \%$ \\
\hline Stovepipe Service & not present & $S$ & $\mathrm{n} / \mathrm{a}$ & $0.081 \mathrm{~s}$ & $\begin{array}{l}{[0 / 0]} \\
100 \%\end{array}$ & $\begin{array}{l}0 / 0] \\
100 \%\end{array}$ & $100 \%$ \\
\hline
\end{tabular}

A4. Performance: The computation time required for the detection of antipatterns using the generated algorithms is reasonably very low, i.e., in the order of few seconds. We perform all experiments on an Intel Dual Core at $3.30 \mathrm{GHz}$ with $4 \mathrm{~GB}$ of RAM. Computation times include computing metric values, introspection delay during static and dynamic analyses, and applying detection algorithms, and do not include the time while targeted services are processing or the time belonging to availability or response time metrics. The computation times for the detection of antipatterns in Home-Automation is reasonably low, i.e., ranging from 0.194s to $1.154 \mathrm{~s}$ with an average of $0.387 \mathrm{~s}$ (see Table 4 ).

Moreover, even for a large scale SBS like FraSCAti, we record a bit lower detection time, i.e., ranging from $0.056 \mathrm{~s}$ to $0.302 \mathrm{~s}$ with an average of $0.089 \mathrm{~s}$. Given a larger 
SBS, the detection time for FraSCAti should be higher than Home-Automation. However, we improved the SOFA implementation since our earlier work [4]. Previously in [4], we compute every metric for each antipattern whenever they appear in the rule cards. In this paper, for FraSCAti, we improve the way SOFA compute different metrics. Indeed, we now compute all the metrics only once and use them for various rule cards. This new implementation strategy substantially reduces the detection time.

The detection times depend mostly on computing some static metrics, such as NMD, CPL, COH, or ANIM. Computing those metrics requires thorough inspection of source code and sometimes a high number of pairwise comparisons among method signatures and/or parameters. However, the complexity of our detection algorithms are linear, i.e., $O(\mathrm{n})$, with $\mathrm{n}$ represents the number of rules. Therefore, the cumulative detection times will increase with the number of rules in each rule card, and with the number of antipatterns to be detected in the target system. Such low computation times suggest that SODA could be applied on SBSs even with a larger number of services. Thus, we show that we support the fourth assumption positively.

\subsection{Threats to Validity}

The main threat to the validity of our results in [4] concerned their external validity, i.e., the possibility to generalise our approach to other SBSs. In this paper, we minimize the external validity by experimenting with another large scale SCA system, i.e., FraSCAti. As a part of the future work, we plan to experiment with other SBSs, if available. For internal validity, the detection results depend on the services provided by the SOFA framework, and also on the antipattern specifications using rule cards. We performed experiments on a representative set of antipatterns to lessen this threat to the internal validity. The subjective nature of specifying and validating antipatterns is a threat to construct validity. We try to lessen this threat by defining rule cards based on thorough literature review and domain analysis, and by involving an independent engineer and the FraSCAti team in the validation. We minimize reliability validity by automating the generation of the detection algorithms, where each subsequent detection produce consistent sets of results with anticipated precision and recall.

\section{Conclusion and Future Work}

The specification and detection of SOA antipatterns are important to assess the design and QoS of SBSs and thus ease the maintenance and evolution of SBSs. In this paper, we presented a novel approach, named SODA, for the specification and detection of SOA antipatterns, and SOFA, its underlying framework. We proposed a DSL for specifying SOA antipatterns and a process for automatically generating detection algorithms from the antipattern specifications. We applied and validated 
SODA with 10 different SOA antipatterns on two SCA systems developed independently: (i) an original and an evolved version of Home-Automation with 13 services and (ii) FraSCAti, an open-source implementation of the SCA standard containing more than 100 services (almost 10 times bigger than Home-Automation). We demonstrated the usefulness of our approach and discussed its precision and recall.

As future work, we want to explore other approaches for detecting SOA antipatterns, i.e., we may analyse execution traces of SBSs. We also intend to enhance the detection approach with a correction approach to suggest refactorings, and automatically, at runtime, correct detected SOA antipatterns, enabling software engineers to improve the design and QoS of their SBSs. Furthermore, we intend to perform other experiments on different SBSs from different SOA technologies, including Web Services, REST and EJB. The approach may require some adaptations from one technology to another because although SOA technologies share some common concepts and principles, they also have their own specific characteristics and implementation styles. Another targeted SBS is the SOFA framework itself since this SBS will certainly evolve to handle various antipatterns and SBSs. We will thus ensure that the evolution of the SOFA framework itself does not introduce any service-oriented antipatterns.

\section{Acknowledgments.}

The authors thank Yousri Kouki and Mahmoud Ben Hassine for their help with respectively an early prototype of SOFA and the implementation of HomeAutomation. The authors are also thankful to the FraSCAti development team and Benjamin Joyen Conseil for validating the results. This work is partly supported by the NESSOS European Network of Excellence and a NSERC Discovery Grant. And, this work is in memory of Anne-Françoise Le Meur, our dearly departed colleague, who initiated the work.

\section{Bibliography}

[1] Thomas Erl. Service-Oriented Architecture: Concepts, Technology, and Design. Prentice Hall PTR, August 2005.

[2] George Spanoudakis and Khaled Mahbub. Requirements Monitoring for Servicebased Systems: Towards a Framework based on Event Calculus. In Automated Software Engineering, 2004. Proceedings. 19th International Conference on, pages 379384,2004

[3] Jaroslav Král and Michal Žemlička. Crucial Service-Oriented Antipatterns. volume 2, pages 160-171. International Academy, Research and Industry Association (IARIA), 2008.

[4] Naouel Moha, Francis Palma, Mathieu Nayrolles, Benjamin Joyen Conseil, Yann-Gaël Guéhéneuc, Benoit Baudry, and Jean-Marc Jézéquel. Specification and Detection of SOA Antipatterns. 10th International Conference on Service Oriented Computing, November 2012.

[5] Lionel Seinturier, Philippe Merle, Damien Fournier, Valerio Schiavoni, Christophe 
Demarey, Nicolas Dolet, and Nicolas Petitprez. FraSCAti - Open SCA Middleware Platform v1.4. http://frascati.ow2.org/.

[6] Lionel Seinturier, Philippe Merle, Romain Rouvoy, Daniel Romero, Valerio Schiavoni, and Jean-Bernard Stefani. A Component-Based Middleware Platform for Reconfigurable Service-Oriented Architectures. Softw. Pract. Exper., 42(5):559-583, May 2012 .

[7] Thomas Erl. SOA Design Patterns. Prentice Hall PTR, January 2009.

[8] Robert Daigneau. Service Design Patterns: Fundamental Design Solutions for SOAP/WSDL and RESTful Web Services. Addison-Wesley, November 2011.

[9] Arnon Rotem-Gal-Oz, Eric Bruno, and Udi Dahan. SOA Patterns. Manning Publications Co., 2012. To be published in Summer 2012.

[10] Bipin Upadhyaya, Ran Tang, and Ying Zou. An Approach for Mining Service Composition Patterns from Execution Logs. Journal of Software: Evolution and Process, 2012.

[11] Qianhui Althea Liang, Jen-Yao Chung, Steven Miller, and Yang Ouyang. Service Pattern Discovery of Web Service Mining in Web Service Registry-Repository. In Proceedings of the IEEE International Conference on e-Business Engineering, ICEBE '06, pages 286-293, Washington, DC, USA, 2006. IEEE Computer Society.

[12] A. J. M. M. Weijters and W. M. P. van der Aalst. Rediscovering Workflow Models from Event-based Data using Little Thumb. Integrated Computer-Aided Engineering, 10(2):151-162, April 2003.

[13] Remco Dijkman, Marlon Dumas, Luciano García-Bañuelos, and Reina Käärik. Aligning Business Process Models. In IEEE International Enterprise Distributed Object Computing Conference, pages 45-53, September 2009.

[14] Mirjam Minor, Alexander Tartakovski, and Ralph Bergmann. Representation and Structure-Based Similarity Assessment for Agile Workflows. In Proceedings of the 7th international conference on Case-Based Reasoning: Case-Based Reasoning Research and Development, ICCBR '07, pages 224-238, Berlin, Heidelberg, 2007. SpringerVerlag.

[15] Luba Cherbakov, Mamdouh Ibrahim, and Jenny Ang. SOA Antipatterns: The Obstacles to the Adoption and Successful Realization of Service-Oriented Architecture. www.ibm.com/developerworks/webservices/library/ws-antipatterns/.

[16] Steve Jones. SOA Anti-patterns. www.infoq.com/articles/SOA-anti-patterns, June 2006.

[17] Tarak Modi. SOA Management: SOA Antipatterns. www.ebizq.net/topics/soa_management/features/7238.html, August 2006.

[18] Bill Dudney, Stephen Asbury, Joseph K. Krozak, and Kevin Wittkopf. J2EE AntiPatterns. John Wiley \& Sons Inc, August 2003.

[19] Nikola Trčka, Wil M. Aalst, and Natalia Sidorova. Data-Flow Anti-patterns: Discovering Data-Flow Errors in Workflows. In Proceedings of the 21st International Conference on Advanced Information Systems Engineering, CAMISE '09, pages 425-439, Berlin, Heidelberg, 2009. Springer-Verlag.

[20] Naouel Moha, Yann-Gaël Guéhéneuc, Laurence Duchien, and Anne-Francoise Le Meur. DECOR: A Method for the Specification and Detection of Code and Design Smells. IEEE Transaction on Software Engineering, 36(1):20-36, January 2010.

[21] Marouane Kessentini, Wael Kessentini, Houari Sahraoui, Mounir Boukadoum, and Ali Ouni. Design Defects Detection and Correction by Example. In IEEE 19th International Conference on Program Comprehension (ICPC), pages 81 -90, june 2011.

[22] Marouane Kessentini, Stéphane Vaucher, and Houari Sahraoui. Deviance From Perfection is a Better Criterion Than Closeness To Evil When Identifying Risky Code. 
In Proceedings of the IEEE/ACM International Conference on Automated Software Engineering, ASE'10, pages 113-122, New York, NY, USA, 2010. ACM.

[23] Michele Lanza and Radu Marinescu. Object-Oriented Metrics in Practice. SpringerVerlag, 2006.

[24] Matthew James Munro. Product Metrics for Automatic Identification of "Bad Smell" Design Problems in Java Source-Code. In Proceedings of the $11^{\text {th }}$ International Software Metrics Symposium. IEEE Computer Society Press, September 2005.

[25] Dimitrios L. Settas, Georgios Meditskos, Ioannis G. Stamelos, and Nick Bassiliades. SPARSE: A Symptom-based Antipattern Retrieval Knowledge-based System using Semantic Web Technologies. Expert Systems with Applications, 38(6):7633-7646, June 2011.

[26] William J. Brown, Raphael C. Malveau, Hays W. "Skip" McCormick, and Thomas J. Mowbray. Anti Patterns: Refactoring Software, Architectures, and Projects in Crisis. John Wiley and Sons, 1998.

[27] Martin J. Fowler, Kent Beck, John Brant, William Opdyke, and Don Roberts. Refactoring: Improving the Design of Existing Code. Addison-Wesley, 1999.

[28] Foutse Khomh, Stephane Vaucher, Yann-Gaël Guéhéneuc, and Houari Sahraoui. BDTEX: A GQM-based Bayesian Approach for the Detection of Antipatterns. Journal of Systems and Software, 84(4):559-572, 2011.

[29] Sunny Wong, Melissa Aaron, Jeffrey Segall, Kevin Lynch, and Spiros Mancoridis. Reverse Engineering Utility Functions Using Genetic Programming to Detect Anomalous Behavior in Software. In Proceedings of the 2010 17th Working Conference on Reverse Engineering, WCRE '10, pages 141-149, Washington, DC, USA, 2010. IEEE Computer Society.

[30] Trevor Parsons and John Murphy. Detecting Performance Antipatterns in Component Based Enterprise Systems. Journal of Object Technology, 7(3):55-90, April 2008.

[31] cbsdetector. Technical report, July 2010.

[32] Bill Pugh, Andrey Loskutov, and Keith Lea. FindBugs. Technical report, December 2012.

[33] Cristina Marinescu, Radu Marinescu, Petru Mihancea, Daniel Ratiu, and Richard Wettel. iPlasma: An Integrated Platform for Quality Assessment of Object-oriented Design. pages 77-80. Society Press, 2005.

[34] JDeodorant. Technical report, Department of Computer Science and Software Engineering, Concordia University.

[35] Vojtech Svatek, Ondrej Svab-Zamazal, Miroslav Vacura, Petr Strossa, Marek Dudáš, Ján Cerný, and Simone Serra. PatOMat. Technical report, Knowledge Engineering Group, University of Economics, Prague, Czech Republic.

[36] Andreas Dangel and Romain Pelisse. PMD. Technical report, last update: May 2013.

[37] SonarSource SA. SonarQube. Technical report, last update: June 2013.

[38] Dimitrios Settas. SPARSE (sparse-antipatt). Technical report, last update: April 2013.

[39] John M. Chambers, William S. Cleveland, Paul A. Tukey, and Beat Kleiner. Graphical Methods for Data Analysis. Wadsworth International, 1983.

[40] Charles Consel and Renaud Marlet. Architecturing Software Using A Methodology for Language Development. Lecture Notes in Computer Science, 1490:170-194, September 1998.

[41] Naouel Moha, Sagar Sen, Cyril Faucher, Olivier Barais, and Jean-Marc Jézéquel. Evaluation of Kermeta for Solving Graph-based Problems. Journal on Software Tools for Technology Transfer, 12(3-4):273-285, July 2010.

[42] David Sciamma, Gilles Cannenterre, and Jacques Lescot. Ecore Tools. Technical re- 
port, last update: May 2013.

[43] Obeo. Acceleo. Technical report, 2005.

[44] William B. Frakes and Ricardo A. Baeza-Yates. Information Retrieval: Data Structures \& Algorithms. Prentice-Hall, 1992. 